\title{
« Monetary policy transparency and inflation persistence in a small open economy »
}

\author{
$\underline{\text { Auteurs }}$ \\ Meixing Dai, Moïse Sidiropoulos, Eleftherios Spyromitros
}

Document de Travail n 2009 - 08

Mars 2009

Faculté des sciences économiques et de gestion

Pôle européen de gestion et d'économie (PEGE) 61 avenue de la Forêt Noire F-67085 Strasbourg Cedex

Secétariat du BETA Géraldine Manderscheidt Tél. : (33) 0390242069 Fax : (33) 0390242070 g.manderscheidt@unistra.fr http://cournot2.u-strasbg.fr/beta 


\title{
Monetary policy transparency and inflation persistence in a small open economy
}

\author{
Meixing DAI ${ }^{\mathrm{a}}$ \\ Moïse SIDIROPOULOS ${ }^{b}$ \\ Eleftherios SPYROMITROS ${ }^{\mathrm{c}}$
}

\begin{abstract}
Using a New Keynesian small open economy model, we examine the effects of central bank transparency on inflation persistence. We have found that more opacity could reinforce the effect of persistent shocks on the level and variability of endogenous variables if the difference between the interest elasticity of domestic goods demand and the degree of trade openness is sufficiently large or sufficiently low, judging on structural parameters characterising the economy, the central bank preference and its initial degree of opacity. Our result implies that, under perfect capital mobility, a high degree of domestic financial development is a good reason for increasing transparency.
\end{abstract}

Key words: Central bank’s transparency, open economy, inflation persistence.

JEL classification numbers: E52, E58, F41.

Corresponding addresses:

${ }^{\text {a }}$ Université de Strasbourg, BETA, 61, avenue de la Forêt Noire - 67085 Strasbourg Cedex - France ; Tel (33) 03 90242131 ; Fax (33) 0390242071 ; e-mail : dai@cournot.u-strasbg.fr.

b LEAP, Department of Economics, Aristotle University of Thessaloniki, Thessaloniki, Greece 54124, E-mail: msidiro@econ.auth.gr, Phone: (30) 23109987 10; and Université de Strasbourg, BETA, 61, avenue de la Forêt Noire - 67085 Strasbourg Cedex - France ; Tel (33) 03902420 85; Fax (33) 0390242071 ; e-mail: sidiro@cournot.u-strasbg.fr.

c Université de Strasbourg, BETA, 61, avenue de la Forêt Noire - 67085 Strasbourg Cedex - France ; Tel (33) 0390 2420 94; Fax (33) 03902420 71 ; e-mail: spyro@cournot.u-strasbg.fr. 


\section{Introduction}

Over the last decade, inflation persistence has become one of the most intensely investigated topics in macroeconomics. This is explained by the crucial role of inflation dynamics on monetary policy design and its effectiveness. Several microeconomic interpretations are proposed to remedy the inability of New Keynesian Phillips curve models to replicate the high inflation persistence found in post-war U.S. data ${ }^{1}$. Researchers have proposed different mechanisms to build inflation persistence into the deep structure of the economy, thus making it invariant to changes in the monetary regime (Buiter and Jewitt (1989), Fuhrer and Moore (1995), Fuhrer (2000), Calvo et al. (2001), Christiano et al. (2005), Blanchard and Gali (2007)). Another approach is to assume that private agents face information-processing constraints (Roberts (1998), Ball (2000), Ireland (2000), Mankiw and Reis (2001), Sims (2001), Woodford (2001), Steinsson (2003)). Some other authors generate inflation persistence through the data generating process for the structural shocks hitting the economy (Rotemberg and Woodford (1997), Ireland (2004), Dittmar et al. (2005)).

An alternative view is that the degree of inflation persistence is not an inherent structural characteristic of industrial economies, but rather varies with the stability and transparency of the monetary policy regime (Sargent, 1999; Goodfriend and King, 2001). In particular, a group of models has emerged attempting to improve the empirical fit of the New Keynesian framework by augmenting it with imperfect credibility and learning (Huh and Lansing (2000), Erceg and Levin (2003), Andolfatto and Gomme (2003), Gaspar et al. (2006)). This literature asserts that the persistence puzzle arises because of the empirically questionable assumption of perfect policy transparency, and not because of any intrinsic shortcomings of the contract structure. The learning by the public of the intentions of the monetary authorities through observing real outcomes is likely to generate additional persistence in inflation dynamics. Criticizing this approach, Westelius (2005) questions, by eliminating discretionary monetary policy in favour of an appropriately specified Taylor rule, how much of the persistence is generated endogenously through imperfect credibility and transparency and how much is exogenously assumed.

\footnotetext{
1 A large empirical literature has found that inflation in developed countries exhibits very high persistence, approaching that of a random-walk process. Fuhrer and Moore (1995) are the first to show that the New Keynesian Phillips curve derived from overlapping wage contract fails to account for the observed persistence in inflation. See also Stock (2001) and Pivetta and Reis (2007).
} 
In an empirical study, Van der Cruijsen and Demertzis (2007), using the transparency measures of Eijffinger and Geraats (2006), have shown that more transparency is also associated with less inflation persistence. Dincer and Eichengreen (2007) confirm the negative relationship between transparency and inflation persistence by extending the transparency data set to more countries and a longer time period. Considering that inflation is set in a more backward looking manner, Eijffinger et al. (2008) find empirical support for an optimal intermediate degree of transparency at which inflation persistence is minimized.

Some studies investigate the effect of transparency in taking account of openness of modern economies. Dillén and Nilsson (1998), using model simulation, show that the convergence to the central bank's inflation target is somewhat faster when transparency is increased and that implies a smaller drift in nominal variables such as the exchange rate. Moreover, there is a clear reduction of variability in real variables such as the real exchange rate and the output gap. Kenneth and Posen (2001), assessing the importance of domestic inflation and interest rate shocks to G3 exchange rate volatility, find that increases in central bank transparency could meaningfully but not totally diminish that volatility. Empirical study by Chortareas et al. (2002) shows significant negative relationship between transparency and inflation, in particular, in countries adopting flexible exchange rate regime. Dincer and Eichengreen (2007) have found empirically that countries with more flexible exchange rates tends to be more transparent in the conduct of monetary policy, explained by the fact that the absence of an exchange rate peg eliminates one traditional device for monitoring central bank actions.

The objective of our paper is to re-examine the persistence puzzle by focusing on the inflation-targeting regime in a New Keynesian open economy model without backward-looking component, where inflation persistence is introduced through the data generating process for the structural shocks hitting the economy. Under flexible exchange rate regime, we study how political transparency affects inflation, output gap and exchange rate dynamics, and evaluate how the effect of opacity depends on the structural parameters of the economy, such as domestic financial development and international trade openness.

Our modelling choices are justified by recent developments in the literature of inflation persistence as well as on international financial markets. Under inflation targeting, i.e. discretionary monetary policy without inflationary bias, Benati (2008) has shown that inflation appears to be (nearly) purely forward-looking, so that no mechanism introducing backward- 
looking components is necessary to fit the data. Current developments on international financial markets have shown that there are considerable persistent real or nominal shocks reflected by the large speculative movements in the prices of oil and other raw materials as well as in exchange rates between major currencies. These shocks tend to feed progressively the inflationary processes, hence constituting great challenge for inflation targeting regimes, in particular when they are not sufficiently transparent about the preferences for output and inflation stabilisation.

Our study is connected to the large literature assessing theoretically and empirically the passthrough of exchange rate shocks to domestic prices and hence inflation dynamics (see among others, Dornbusch (1987) and Krugman (1987), Goldberg and Knetter (1997), Mennon (1995), Smets and Wouters (2002), Devereux and Engel (2002), Corsetti et al. (2008)). Our study contributes to this literature by studying how the political transparency of the central bank affects the pass-through of exchange rate shocks to the rest of the economy, in particular to the inflation dynamics. In view of the large movements of exchange rates under floating exchange rate regime, the pass-through of exchange rate shocks to domestic prices, and hence inflation and inflation expectations is of particular importance for monetary policy. Both the size of the passthrough and its speed are essential for the proper assessment of the monetary policy transmission to prices as well as for inflation forecasts.

The paper is structured as follows. Section 2 presents the model and introduces incomplete information about the central bank preferences. Section 3 gives the equilibrium solutions of endogenous variables. Section 4 analyzes the nature of the relationship between monetary policy transparency and inflation, output gap and real exchange rate persistence. Section 5 examines the effect of transparency on macroeconomic volatility. Section 6 concludes.

\section{The small open-economy model}

We use a stylized New Keynesian model of a small open economy² developed by Galí and Monacelli (2005) and Clarida et al. (2001) and introduce a time-varying premium on foreign bond holdings as in Leitemo and Söderström (2008), which is an important source of uncertainty in open economies.

2 The model is a generalization of the canonical New Keynesian model for a closed economy developed by Rotemberg and Woodford (1997), Goodfriend and King (1997) and others, and carefully examined by Clarida et al. (1999). 
The small open home country trades with the rest of the world which is assumed to be a large foreign country. The micro-foundations of this model are based on the assumption that the two countries share preferences and technology and produce traded consumption goods. Concerning the home country, domestic firms use only labour to produce goods, and households consume both domestically produced and imported goods and enjoy leisure.

The (log) real exchange rate, $e_{t}$, is defined in terms of the domestic price level as

$$
e_{t}=s_{t}+p_{t}^{f}-p_{t},
$$

where $s_{t}$ is the nominal exchange rate, $p_{t}^{f}$ is the price level in the foreign economy, and $p_{t}$ is the price level of domestically produced goods.

The rate of inflation in the domestic goods sector, $\pi_{t}$, the output gap in the domestic economy (i.e., the log deviation of domestic output from its flexible-price level), $x_{t}$, and the (log) real exchange rate are interrelated according to the following three equations:

$$
\begin{aligned}
& \pi_{t}=\beta \mathrm{E}_{t} \pi_{t+1}+\delta x_{t}+\phi e_{t}+\varepsilon_{t}^{\pi}, \quad \text { with } 0<\beta<1,0<\phi<1, \delta>0, \\
& x_{t}=E_{t} x_{t+1}-\alpha\left(i_{t}-E_{t} \pi_{t+1}\right)+\gamma\left(E_{t} e_{t+1}-e_{t}\right)+\varepsilon_{t}^{x}, \quad \text { with } \alpha, \gamma>0, \\
& e_{t}=E_{t} e_{t+1}-\left(i_{t}-E_{t} \pi_{t+1}\right)+\varepsilon_{t}^{e},
\end{aligned}
$$

Equation (2) represents the open-economy New Keynesian Phillips curve, where the domestic inflation rate depends on expected future inflation $\left(\mathrm{E}_{t} \pi_{t+1}\right)$ and current marginal cost, which is affected by the output gap and the real exchange rate. In effect, the latter affects marginal cost through the labour supply decision of households which value their wage relative to the consumer price index including prices of imported goods. The inflation shock, $\varepsilon_{t}^{\pi}$, is due to productivity disturbances.

Equation (3), an expectational IS curve, relates the output gap to the expected future output gap $\left(E_{t} x_{t+1}\right)$, the real interest rate and the real exchange rate. The real interest rate is defined as the difference between the nominal interest rate $\left(i_{t}\right)$ and the expected future inflation. The demand shock, $\varepsilon_{t}^{x}$, reflects either productivity disturbances which affect the flexible-price level of output or, equivalently, changes in the natural real interest rate.

Finally, equation (4) is a real uncovered interest rate parity (UIP) condition, where the expected rate of real depreciation $\left(E_{t} e_{t+1}-e_{t}\right)$ is related to the real interest rate differential 
between the domestic and foreign economies. Since all foreign variables are assumed to be exogenous and set to zero, they are absent in this equation as well as in the rest of the model. The exchange rate disturbance, $\varepsilon_{t}^{e}$, stands for a risk premium shock on foreign bond holding.

We assume that all disturbances to the economy follow a first-order autoregressive process:

$$
\varepsilon_{t}^{j}=\rho_{j} \varepsilon_{t-1}^{j}+e_{t}^{j}, \quad \text { with } j=\pi, x, e, \quad 0 \leq \rho_{j} \leq 1 ;
$$

where $\rho_{j}$ represents the degree of persistence.

The model is completed with a specification of the central bank's loss function:

$$
L^{C B}=\frac{1}{2} E_{0} \sum_{t=0}^{\infty} \beta^{i}\left(\lambda x_{t}^{2}+\pi_{t}^{2}\right) .
$$

The central bank's loss depends on output gap and inflation variability around zero targets. The central bank can be more or less transparent in the disclosure of information about its preferences, i.e. the relative weight $(\lambda>0)$ assigned to the output-gap objective. This corresponds to political transparency in the terminology given in Geraats (2002). In the following, we define complete transparency of monetary policy as a benchmark situation where the central bank communicates the exact value of $\lambda$ to the public, in other words, the variance of $\lambda$ is zero $\left(\sigma_{\lambda}^{2}=0\right)$. In this case, the expected value of $\lambda$, denoted by $E_{t}(\lambda)=\bar{\lambda}$, is equal to its realized value, i.e. $\bar{\lambda}=\lambda$. When the variance of $\lambda$ is superior to zero $\left(\sigma_{\lambda}^{2}>0\right)$ and increases, central bank transparency decreases or alternatively central bank opacity increases. In the presence of opacity, the expected value of $\lambda$ could be different from the true one, i.e. $\bar{\lambda} \neq \lambda$. As we will show below, the opacity as well as preference perception error have important implications for monetary policy decision-making.

The central bank minimizes its loss function (6) taking account of the economic model defined by equations (2)-(4). The Lagrangian for this problem is given by:

$$
\Lambda=E_{0} \sum_{t=0}^{\infty}\left\{\begin{array}{l}
\frac{1}{2}\left(\lambda x_{t}^{2}+\pi_{t}^{2}\right)-\mu_{t}^{\pi}\left[\pi_{t}-\beta \mathrm{E}_{t} \pi_{t+1}-\delta x_{t}-\phi e_{t}-\varepsilon_{t}^{\pi}\right] \\
-\mu_{t}^{x}\left[x_{t}-E_{t} x_{t+1}+\alpha\left(i_{t}-E_{t} \pi_{t+1}\right)-\gamma\left(E_{t} e_{t+1}-e_{t}\right)-\varepsilon_{t}^{x}\right] \\
-\mu_{t}^{e}\left[e_{t}-E_{t} e_{t+1}+\left(i_{t}-E_{t} \pi_{t+1}\right)-\varepsilon_{t}^{e}\right]
\end{array}\right\},
$$

where $\mu_{t}^{j}, j=\pi, x, e$, are Lagrange multipliers associated with equations (2)-(4) respectively. The first-order conditions for this minimisation problem are: 


$$
\begin{aligned}
& \frac{\partial \Lambda}{\partial \pi_{t}}=\pi_{t}-\mu_{t}^{\pi}=0, \\
& \frac{\partial \Lambda}{\partial x_{t}}=\lambda x_{t}+\delta \mu_{t}^{\pi}-\mu_{t}^{X}=0, \\
& \frac{\partial \Lambda}{\partial i_{t}}=-\mu_{t}^{x} \alpha-\mu_{t}^{e}=0, \\
& \frac{\partial \Lambda}{\partial e_{t}}=-\mu_{t}^{x} \gamma-\mu_{t}^{e}=0 .
\end{aligned}
$$

From condition (8), it follows that $\mu_{t}^{\pi}=\pi_{t}$. Using conditions (10) and (11), we obtain $(\gamma-\alpha) \mu_{t}^{x}=0$. Assuming that $\alpha \neq \gamma$, then we get $\mu_{t}^{x}=0$ and consequently $\mu_{t}^{e}=0$. Using these results in condition (9), we get the targeting rule in the sense of Svensson (2002):

$$
x_{t}=-\frac{\delta}{\lambda} \pi_{t} .
$$

This rule is also valid for the next period, hence we have:

$$
E_{t} X_{t+1}=-\frac{\delta}{\lambda} E_{t} \pi_{t+1}
$$

Using equations (2)-(4) and (12)-(13), we obtain the instrument rule in the sense of Svensson (2002):

$$
i_{t}=E_{t} \pi_{t+1}+\frac{\left(\lambda \beta-1-\delta^{2}\right) \delta}{\lambda\left[\delta \phi+\left(1+\delta^{2}\right)(\alpha-\gamma)\right]} E_{t} \pi_{t+1}+\frac{\delta \phi}{\delta \phi+\left(1+\delta^{2}\right)(\alpha-\gamma)} E_{t} e_{t+1}+\frac{\left(1+\delta^{2}\right) \varepsilon_{t}^{x}+\left[\delta \phi-\left(1+\delta^{2}\right) \gamma\right] \varepsilon_{t}^{e}+\delta \varepsilon_{t}^{\pi}}{\delta \phi+\left(1+\delta^{2}\right)(\alpha-\gamma)} .
$$

The optimal interest rate reacts positively to the expected future inflation if $\alpha-\gamma>\max \left\{-\frac{\delta \phi}{1+\delta^{2}} ;-\frac{\left(\lambda \beta-1-\delta^{2}\right) \delta}{\lambda\left(1+\delta^{2}\right)}-\frac{\delta \phi}{\left(1+\delta^{2}\right)}\right\} \quad$ or $\quad \alpha-\gamma<\min \left\{-\frac{\delta \phi}{1+\delta^{2}} ;-\frac{\left(\lambda \beta-1-\delta^{2}\right) \delta}{\lambda\left(1+\delta^{2}\right)}-\frac{\delta \phi}{1+\delta^{2}}\right\}$. It reacts positively to the expected future real exchange rate as well as $\varepsilon_{t}^{x}$ and $\varepsilon_{t}^{\pi}$ if $\alpha-\gamma>-\frac{\delta \phi}{1+\delta^{2}}$, and positively to $\varepsilon_{t}^{e}$ if $\gamma<\frac{\delta \phi}{1+\delta^{2}}$ or $\alpha+\frac{\delta \phi}{1+\delta^{2}}<\gamma$.

\section{Equilibrium solutions under persistent shocks}

Using equations (3)-(4) and (12)-(14), we obtain:

$$
E_{t} e_{t+1}=\frac{\beta \lambda(\alpha-\gamma)+\phi \delta}{\beta \lambda(\alpha-\gamma)} e_{t}+\frac{\left[\lambda \beta-\left(1+\delta^{2}\right)\right] \delta}{\beta \lambda^{2}(\alpha-\gamma)} \pi_{t}+\frac{1}{\alpha-\gamma} \varepsilon_{t}^{X}-\frac{\alpha}{\alpha-\gamma} \varepsilon_{t}^{e}+\frac{\delta}{\beta \lambda(\alpha-\gamma)} \varepsilon_{t}^{\pi},
$$




$$
E_{t} \pi_{t+1}=\frac{1+\delta^{2}}{\beta \lambda} \pi_{t}-\frac{\phi}{\beta} e_{t}-\frac{1}{\beta} \varepsilon_{t}^{\pi} .
$$

Under the rational expectations hypothesis, we solve endogenous variables using the method of undetermined coefficients (McCallum, 1983). In the following, we focus on the solutions of $\pi_{t}, E_{t} \pi_{t+1}, e_{t}$ and $E_{t} e_{t+1}$. The solutions of other endogenous variables, $x_{t}, E_{t} x_{t+1}$ and $i_{t}$, can be obtained using the solutions of $\pi_{t}, E_{t} \pi_{t+1}, e_{t}$ and $E_{t} e_{t+1}$, and equations (12)-(14). In accordance with the method of undetermined coefficients, we guess that the solutions of $\pi_{t}, e_{t}, E_{t} \pi_{t+1}$ and $E_{t} e_{t+1}$ take the following forms:

$$
\begin{aligned}
& \pi_{t}=\zeta_{1} \varepsilon_{t}^{\pi}+\zeta_{2} \varepsilon_{t}^{e}+\zeta_{3} \varepsilon_{t}^{x}, \\
& e_{t}=\chi_{1} \varepsilon_{t}^{\pi}+\chi_{2} \varepsilon_{t}^{e}+\chi_{3} \varepsilon_{t}^{x}, \\
& \mathrm{E}_{t} \pi_{t+1}=\mathrm{E}_{t} \zeta_{1} \mathrm{E}_{t} \varepsilon_{t+1}^{\pi}+\mathrm{E}_{t} \zeta_{2} \mathrm{E}_{t} \varepsilon_{t+1}^{e}+\mathrm{E}_{t} \zeta_{3} \mathrm{E}_{t} \varepsilon_{t+1}^{x}, \\
& \mathrm{E}_{t} e_{t+1}=\mathrm{E}_{t} \chi_{1} \mathrm{E}_{t} \varepsilon_{t+1}^{\pi}+\mathrm{E}_{t} \chi_{2} \mathrm{E}_{t} \varepsilon_{t+1}^{e}+\mathrm{E}_{t} \chi_{3} \mathrm{E}_{t} \varepsilon_{t+1}^{x},
\end{aligned}
$$

where $\zeta_{i}$ and $\chi_{i}, i=1,2,3$, are coefficients that we seek to determine in order to find the equilibrium solutions of $\pi_{t}, E_{t} \pi_{t+1}, e_{t}$ and $E_{t} e_{t+1}$. Using equation (5), equations (19) and (20) are rewritten as:

$$
\begin{aligned}
& \mathrm{E}_{t} \pi_{t+1}=\mathrm{E}_{t} \zeta_{1} \rho_{\pi} \varepsilon_{t}^{\pi}+\mathrm{E}_{t} \zeta_{2} \rho_{e} \varepsilon_{t}^{e}+\mathrm{E}_{t} \zeta_{3} \rho_{x} \varepsilon_{t}^{x}, \\
& \mathrm{E}_{t} e_{t+1}=\mathrm{E}_{t} \chi_{1} \rho_{\pi} \varepsilon_{t}^{\pi}+\mathrm{E}_{t} \chi_{2} \rho_{e} \varepsilon_{t}^{e}+\mathrm{E}_{t} \chi_{3} \rho_{x} \varepsilon_{t}^{x} .
\end{aligned}
$$

Substituting $\mathrm{E}_{t} \pi_{t+1}$ and $\mathrm{E}_{t} e_{t+1}$ given by equations (21)-(22) into equations (15)-(16), we obtain:

$$
\begin{aligned}
& \mathrm{E}_{t} \zeta_{1} \rho_{\pi} \varepsilon_{t}^{\pi}+\mathrm{E}_{t} \zeta_{2} \rho_{e} \varepsilon_{t}^{e}+\mathrm{E}_{t} \zeta_{3} \rho_{X} \varepsilon_{t}^{x}=\frac{1+\delta^{2}}{\beta \lambda} \pi_{t}-\frac{\phi}{\beta} e_{t}-\frac{1}{\beta} \varepsilon_{t}^{\pi}, \\
& \mathrm{E}_{t} \chi_{1} \rho_{\pi} \varepsilon_{t}^{\pi}+\mathrm{E}_{t} \chi_{2} \rho_{e} \varepsilon_{t}^{e}+\mathrm{E}_{t} \chi_{3} \rho_{X} \varepsilon_{t}^{x}=\frac{\beta \lambda(\alpha-\gamma)+\phi \delta}{\beta \lambda(\alpha-\gamma)} e_{t}+\frac{\left[\lambda \beta-\left(1+\delta^{2}\right)\right] \delta}{\beta \lambda^{2}(\alpha-\gamma)} \pi_{t}+\frac{\beta \lambda \varepsilon_{t}^{X}-\alpha \beta \lambda \varepsilon_{t}^{e}+\delta \varepsilon_{t}^{\pi}}{\beta \lambda(\alpha-\gamma)} .
\end{aligned}
$$

Solving equations (23)-(24) for $\pi_{t}$ and $e_{t}$, and comparing these solutions with equations (17)(18), we obtain equations relating undetermined coefficients $\zeta_{i}$ and $\chi_{i}$ and their expected value. Solving them, taking account of the opacity about the central bank preferences, leads to: ${ }^{3}$

$$
\zeta_{1}=\Psi\left\{(\bar{\lambda}-\lambda) \phi \delta(\alpha-\gamma) \bar{\lambda}^{3}\left(1-\rho_{\pi}\right) \rho_{\pi}+\lambda(\alpha-\gamma) \bar{\lambda}^{3}\left(1-\rho_{\pi}\right)\left[(\alpha-\gamma)\left(1+\delta^{2}\right)+\phi \delta\right]\right\}
$$

\footnotetext{
3 We use a second-order Taylor development to evaluate the effect of opacity. The details of solutions can be obtained upon request addressed to authors.
} 


$$
\begin{aligned}
& \chi_{1}=\frac{\Psi \delta \bar{\lambda}}{\lambda}\left\{\begin{array}{l}
\bar{\lambda}^{2}\left[\left(1+\delta^{2}\right)(\alpha-\gamma)\left(\bar{\lambda} \rho_{\pi}-\lambda\right)-\lambda \delta \phi\left(1-\rho_{\pi}\right)\right] \\
+\left(1+\delta^{2}\right)(\alpha-\gamma)\left[\bar{\lambda}^{2}(\lambda-\bar{\lambda})+\lambda \sigma_{\lambda}^{2}\right] \rho_{\pi}^{2}
\end{array}\right\}, \\
& \mathrm{E}_{t} \zeta_{1}=-\Psi \bar{\lambda}(\alpha-\gamma) \bar{\lambda}^{3}\left\{\phi \rho_{\pi} \delta+\left(1+\delta^{2}\right)(\alpha-\gamma) \rho_{\pi}-\left[(\alpha-\gamma)\left(1+\delta^{2}\right)+\phi \delta\right]\right\}, \\
& \mathrm{E}_{t} \chi_{1}=\Psi \delta \bar{\lambda}\left\{\begin{array}{l}
{\left[\left(1+\delta^{2}-\bar{\lambda} \beta\right) \bar{\lambda}^{2}+\left(1+\delta^{2}\right) \sigma_{\lambda}^{2}\right](\alpha-\gamma) \rho_{\pi}} \\
+\bar{\lambda}^{2}\left\{[\beta \bar{\lambda}(\alpha-\gamma)+\phi \delta] \rho_{\pi}-\left[(\alpha-\gamma)\left(1+\delta^{2}\right)+\phi \delta\right]\right\}
\end{array}\right\}, \\
& \zeta_{2}=-\alpha \zeta_{3}=\Omega \alpha \phi \bar{\lambda}^{2}\left\{(\bar{\lambda}-\lambda) \delta \phi \rho_{e}+\lambda\left[(\alpha-\gamma)\left(1+\delta^{2}\right)+\phi \delta\right]\right\}, \\
& \chi_{2}=-\alpha \chi_{3}=\frac{\Omega \alpha \bar{\lambda}^{2}}{\lambda}\left\{\left(1+\delta^{2}\right)(\bar{\lambda}-\lambda) \delta \phi \rho_{e}+\lambda\left[\left(1+\delta^{2}\right)-\beta \bar{\lambda} \rho_{e}\right]\left[(\alpha-\gamma)\left(1+\delta^{2}\right)+\phi \delta\right]\right\}, \\
& \mathrm{E}_{t} \zeta_{2}=-\alpha \mathrm{E}_{t} \zeta_{3}=\Omega\left[(\alpha-\gamma)\left(1+\delta^{2}\right)+\phi \delta\right] \alpha \phi \bar{\lambda}^{3}, \\
& \mathrm{E}_{t} \chi_{2}=-\alpha \mathrm{E}_{t} \chi_{3}=\Omega\left\{\left[\left(1+\delta^{2}\right)(\alpha-\gamma)+\phi \delta\right]\left[\left(1+\delta^{2}\right)-\bar{\lambda} \beta \rho_{e}\right] \alpha \bar{\lambda}^{2}+\left(1+\delta^{2}\right) \phi \alpha \delta \rho_{e} \sigma_{\lambda}^{2}\right\},
\end{aligned}
$$

where

$$
\begin{aligned}
& \Psi=\left\{\begin{array}{l}
-\delta\left[\left(1+\delta^{2}-\bar{\lambda} \beta\right) \bar{\lambda}^{2}+\delta\left(1+\delta^{2}\right) \sigma_{\lambda}^{2}\right] \bar{\lambda}(\alpha-\gamma) \phi \rho_{\pi}^{2}+ \\
+\bar{\lambda}^{3}\left\{\left(1+\delta^{2}\right)(\alpha-\gamma) \rho_{\pi}-\left[(\alpha-\gamma)\left(1+\delta^{2}\right)+\phi \delta\right]\right\}\left\{[\beta \bar{\lambda}(\alpha-\gamma)+\phi \delta] \rho_{\pi}-(\alpha-\gamma)\left(1+\delta^{2}\right)-\phi \delta\right\}
\end{array}\right\}^{-1}, \\
& \Omega=\left\{\begin{array}{l}
\left\{\left[(\alpha-\gamma)\left(1+\delta^{2}\right)+\phi \delta\right]-[\beta \bar{\lambda}(\alpha-\gamma)+\phi \delta] \rho_{e}\right\}\left[(\alpha-\gamma)\left(1+\delta^{2}\right)\left(1-\rho_{e}\right)+\phi \delta\right] \bar{\lambda}^{2} \\
-\delta\left(1+\delta^{2}-\bar{\lambda} \beta\right] \lambda^{2} \phi(\alpha-\gamma) \rho_{e}^{2}-\delta\left(1+\delta^{2}\right) \sigma_{\lambda}^{2} \phi(\alpha-\gamma) \rho_{e}^{2}
\end{array}\right.
\end{aligned}
$$

Substituting the solutions of $\zeta_{i}$ and $\chi_{i}$ and their expected value into equations (17)-(20) gives the equilibrium solutions of $\pi_{t}, e_{t}, \mathrm{E}_{t} \pi_{t+1}$ and $\mathrm{E}_{t} e_{t+1}$. It is then easy to obtain the solutions of $x_{t}$ and $E_{t} x_{t+1}$ using equations (12)-(13), and that of $i_{t}$ using equation (14).

We remark in examining the above solutions that if $\alpha=\gamma$, an increase in opacity will have no effect on the level and variability of endogenous variables. In the following, we will study the cases where we have $\alpha \neq \gamma$.

\section{The effect of central bank's opacity on the persistence}

The equilibrium solutions of $\pi_{t}, e_{t}, x_{t}, \mathrm{E}_{t} \pi_{t+1}, \mathrm{E}_{t} e_{t+1}$ and $E_{t} x_{t+1}$ associate the effects of persistent shocks with the degree of central bank opacity. Therefore, they allow us to study how the opacity affects the persistence and variability of endogenous variables. In the following, we will not discuss the effects of opacity on the transmission of persistent shocks to the level and 
variability of $x_{t}$ and $E_{t} x_{t+1}$, since they could be easily deduced from the results concerning $\pi_{t}$ and $\mathrm{E}_{t} \pi_{t+1}$ using equations (12)-(13). We also neglect the effects of $\varepsilon_{t}^{x}$ since equations (29)-(32) show that they are easily deductible from these of $\varepsilon_{t}^{\pi}$.

\subsection{The effects of opacity and the persistence due to inflation shocks}

The effects of persistent inflation and exchange rate shocks are transmitted to the economy through a complex mechanism. The central bank preferences about inflation and output gap targets affect the current inflation and real exchange rate through its effects on nominal interest rate. The perception of the central bank preferences (i.e. the expected value and variance of $\lambda$ ) plays a role in this transmission mechanism through the expectations of inflation and exchange rate. These expectations are taken into account by the central bank in the determination of optimal nominal interest rate according to equation (14), which implies that if the Phillips curve is independent of exchange rate, i.e. $\phi=0$, then the nominal interest rate will only depend on the expected future inflation and exogenous shocks.

Deriving the solutions of $\zeta_{1}, \chi_{1}, \mathrm{E}_{t} \zeta_{1}$ and $\mathrm{E}_{t} \chi_{1}$ given by (25)-(28) with respect to the variance of $\lambda$ gives the effects of opacity on the persistence of $\pi_{t}, e_{t}, \mathrm{E}_{t} \pi_{t+1}$, and $\mathrm{E}_{t} e_{t+1}$ due to persistent inflation shocks. The results are summarized in the following propositions.

Proposition 1a: An increase in opacity reinforces the effects of persistent inflation shocks on the expected future inflation if $\alpha-\gamma>-\frac{\phi \delta}{1+\delta^{2}}$ and vice versa.

Proof: In the presence of persistent inflation shocks, the effect of opacity on expected future inflation is defined as $\frac{\partial \mathrm{E}_{t} \pi_{t+1}}{\partial \sigma_{\lambda}^{2}}=\frac{\rho_{\pi} \partial \mathrm{E}_{t} \zeta_{1}}{\partial \sigma_{\lambda}^{2}}$, which can be examined through deriving $\mathrm{E}_{t} \zeta_{1}$, given by equation (27), with respect to $\sigma_{\lambda}^{2}$ :

$$
\frac{\partial \mathrm{E}_{t} \zeta_{1}}{\partial \sigma_{\lambda}^{2}}=\Psi^{2} \delta \phi \rho_{\pi}^{2} \bar{\lambda}^{5}(\alpha-\gamma)^{2}\left(1+\delta^{2}\right)\left(1-\rho_{\pi}\right)\left[(\alpha-\gamma)\left(1+\delta^{2}\right)+\phi \delta\right] .
$$

It is straightforward to see that: $\frac{\partial \mathrm{E}_{t} \zeta_{1}}{\partial \sigma_{\lambda}^{2}}>0$ if $\alpha-\gamma>\frac{-\phi \delta}{1+\delta^{2}}$ or $\frac{\partial \mathrm{E}_{t} \zeta_{1}}{\partial \sigma_{\lambda}^{2}}<0$ if $\alpha-\gamma<\frac{-\phi \delta}{1+\delta^{2}}$. Q.E.D. 
The opacity affects the expected future inflation and real exchange rate. It influences the macroeconomic performance through several channels which may have contradictory effects. If the Phillips curve is independent of the real exchange rate, i.e. $\phi=0$, the expected future inflation will be determined uniquely by equation (16) independently of other endogenous variables. In this case, an increase in opacity will weaken the effect of persistent inflation shocks on the current and expected future inflation rates (Appendix A).

The introduction of the real exchange rate effect in the Phillips curve induces complex interaction through IS curve and the UIP. In effect, a decrease in the current and expected future inflation rates will induce that of the current and expected future real exchange rates. These variations will induce the following effects: a decrease in the expected future inflation implies an increase in the expected future output gap and a decrease in real interest rate, and therefore an increase in the demand for the domestic goods; a decrease in the current real exchange rate reduces the current inflation rate and increases the demand for domestic goods; a decrease in the expected future real exchange rate reduces the demand for domestic goods. If the positive effects on the domestic goods demand globally dominate the negative ones (i.e. there is an inflationary pressure), the previous negative effect of opacity on the expected future inflation will be reversed. This explains why the relationship between the parameters $\alpha, \gamma, \phi$ and $\delta$ plays an important role in determining the final effects of opacity. In the presence of persistent inflation shocks, more opacity does not increase systematically the inflation expectations and its effects depend on these structural parameters.

We notice that the parameter $\gamma$ describes how the domestic goods demand is impacted by the variation of real exchange rate due to the substitution effects between domestic and foreign goods and hence represents the degree of trade openness; $\alpha$ captures the effect of a variation of real interest rate on the domestic goods demand and hence represents the degree of domestic financial development; $\delta$ represents the slope of the Phillips curve; and the parameter $\phi$ reveals the part of the wage claims which is influenced by the weight of the foreign goods in the consumption of domestic workers, i.e. the effect of real exchange rate on marginal cost.

Proposition 1b: i) An increase in opacity reinforces the effects of persistent inflation shocks on the expected future real exchange rate if one of the following pairs of conditions is checked: 
1) $\bar{\lambda}>\frac{1+\delta^{2}}{\beta \rho_{\pi}}$ and $\alpha-\gamma>\max \left\{0 ; \frac{\phi \delta\left(1-\rho_{\pi}\right)}{\beta \bar{\lambda} \rho_{\pi}-1-\delta^{2}}\right\}$;

2) $\bar{\lambda}>\frac{1+\delta^{2}}{\beta \rho_{\pi}}$ and $\alpha-\gamma<-\frac{\phi \delta}{1+\delta^{2}}$;

3) $\bar{\lambda}<\frac{1+\delta^{2}}{\beta \rho_{\pi}}$ and $\frac{\phi \delta\left(1-\rho_{\pi}\right)}{\beta \bar{\lambda} \rho_{\pi}-1-\delta^{2}}<\alpha-\gamma<-\frac{\phi \delta}{1+\delta^{2}}$;

4) $\bar{\lambda}<\frac{1+\delta^{2}}{\beta \rho_{\pi}}$ and $-\frac{\phi \delta}{1+\delta^{2}}<\alpha-\gamma<\frac{\phi \delta\left(1-\rho_{\pi}\right)}{\beta \bar{\lambda} \rho_{\pi}-1-\delta^{2}}$.

ii) An increase in opacity reduces the effects of persistent inflation shocks on the expected future real exchange rate if one of the following pairs of conditions is checked:
1) $\bar{\lambda}>\frac{1+\delta^{2}}{\beta \rho_{\pi}}$ and $\alpha-\gamma>\frac{\phi \delta\left(1-\rho_{\pi}\right)}{\beta \bar{\lambda} \rho_{\pi}-1-\delta^{2}}$;
2) $\bar{\lambda}>\frac{1+\delta^{2}}{\beta \rho_{\pi}}$ and $-\frac{\phi \delta}{1+\delta^{2}}<\alpha-\gamma<0$;
3) $\bar{\lambda}<\frac{1+\delta^{2}}{\beta \rho_{\pi}}$ and $\max \left\{-\frac{\phi \delta}{1+\delta^{2}} ; \frac{\phi \delta\left(1-\rho_{\pi}\right)}{\beta \bar{\lambda} \rho_{\pi}-1-\delta^{2}}\right\}<\alpha-\gamma<0$;
4) $\bar{\lambda}<\frac{1+\delta^{2}}{\beta \rho_{\pi}}$ and $\alpha-\gamma<\min \left\{\frac{-\phi \delta}{1+\delta^{2}} ; \frac{\phi \delta\left(1-\rho_{\pi}\right)}{\beta \bar{\lambda} \rho_{\pi}-1-\delta^{2}}\right\}$.

Proof: See Appendix B. Q.E.D.

In the conditions specified in the first part of Proposition 1b, we find that, besides the usual conditions on the relationship between $\alpha, \gamma, \phi$ and $\delta$, the estimated value of $\lambda$ plays also a significant role. If $\bar{\lambda}>\frac{1+\delta^{2}}{\beta \rho_{\pi}}$, i.e. the central bank puts in average a relatively high weight on the output target, the opacity reinforces the effect of persistent inflation shocks for relatively high or low value of $\alpha-\gamma$ according to conditions 1 and 2. In other words, if the public estimates that the central bank puts more weight on the output target, more transparency allows reducing the inflation pressure and hence expected future depreciation of domestic currency. In contrast, more opacity will increase the inflation pressure and hence expected future real exchange rate. If $\bar{\lambda}<\frac{1+\delta^{2}}{\beta \rho_{\pi}}$, an increase in opacity reinforces the effect of persistent inflation shocks for intermediate value of $\alpha-\gamma$ according to conditions 3 and 4. Similar comments can be made for the opposite cases enunciated in the second part of Proposition $1 \mathrm{~b}$. 
Proposition 1c: An increase in opacity reinforces the effects of persistent inflation shocks on the current inflation rate if $\alpha-\gamma>-\frac{\phi \delta}{1+\delta^{2}}\left(1-\frac{\bar{\lambda}-\lambda}{\lambda} \rho_{\pi}\right)$, and vice versa.

Proof: The effects of opacity on inflation persistence $\left(\frac{\partial \pi_{t}}{\partial \sigma_{\lambda}^{2}}=\frac{\rho_{\pi} \partial \zeta_{1}}{\partial \sigma_{\lambda}^{2}}\right)$ is obtained by deriving $\zeta_{1}$ given by equation (25) with respect to $\sigma_{\lambda}^{2}$ :

$$
\frac{\partial \zeta_{1}}{\partial \sigma_{\lambda}^{2}}=\Psi^{2}\left\{(\bar{\lambda}-\lambda) \phi \delta \rho_{\pi}+\lambda\left[(\alpha-\gamma)\left(1+\delta^{2}\right)+\phi \delta\right]\right\} \bar{\lambda}^{4} \delta\left(1+\delta^{2}\right)(\alpha-\gamma)^{2} \phi\left(1-\rho_{\pi}\right) \rho_{\pi}^{2} .
$$

Having $\frac{\partial \zeta_{1}}{\partial \sigma_{\lambda}^{2}}>0$ is equivalent to having: $\alpha-\gamma>-\frac{\phi \delta}{1+\delta^{2}}\left(1-\frac{\bar{\lambda}-\lambda}{\lambda} \rho_{\pi}\right)$ Q.E.D.

The condition $\alpha-\gamma>-\frac{\phi \delta}{1+\delta^{2}}\left(1-\frac{\bar{\lambda}-\lambda}{\lambda} \rho_{\pi}\right)$ puts in relation several important parameters of the economy. This condition is more or less restrictive if the perception error $\frac{\bar{\lambda}-\lambda}{\lambda}$ of the central bank preferences is positive or negative respectively. In fact, if $\frac{\bar{\lambda}-\lambda}{\lambda}>0$, it means that the value of $\lambda$ perceived by the private agents is higher than the realized one. In this case, the effect of persistent inflation shocks on the current inflation rate is stronger due to the fact that the private agents expect higher future inflation.

Proposition 1d: An increase in opacity reinforces the effects of persistent inflation shocks on the current real exchange rate if either of the following pairs of conditions is satisfied:

1) $\bar{\lambda}<\frac{1+\delta^{2}}{\beta \rho_{\pi}}$ and $\alpha-\gamma>-\frac{\delta \phi(\bar{\lambda}-\lambda) \rho_{\pi}}{\lambda\left(1+\delta^{2}-\beta \bar{\lambda} \rho_{\pi}\right)}-\frac{\phi \delta}{1+\delta^{2}}$

2) $\bar{\lambda}>\frac{1+\delta^{2}}{\beta \rho_{\pi}}$ and $\alpha-\gamma<-\frac{\delta \phi(\bar{\lambda}-\lambda) \rho_{\pi}}{\lambda\left(1+\delta^{2}-\beta \bar{\lambda} \rho_{\pi}\right)}-\frac{\phi \delta}{1+\delta^{2}}$.

An increase in opacity reduces the effects of persistent inflation shocks on the current real exchange rate if the second inequality in these pairs of conditions is inversed.

Proof: The effects of opacity on the current real exchange rate $\left(\frac{\partial e_{t}}{\partial \sigma_{\lambda}^{2}}=\frac{\rho_{\pi} \partial \chi_{1}}{\partial \sigma_{\lambda}^{2}}\right)$ are obtained by deriving the solution of $\chi_{1}$ given by (26) with respect to $\sigma_{\lambda}^{2}$ as follows: 


$$
\frac{\partial \chi_{1}}{\partial \sigma_{\lambda}^{2}}=\frac{\Psi^{2}\left(1+\delta^{2}\right)(\alpha-\gamma)^{2} \delta \bar{\lambda}^{4} \rho_{\pi}^{2}\left(1-\rho_{\pi}\right)\left\{\left(1+\delta^{2}\right) \delta \phi(\bar{\lambda}-\lambda) \rho_{\pi}+\lambda\left(1+\delta^{2}-\beta \bar{\lambda} \rho_{\pi}\right)\left[(\alpha-\gamma)\left(1+\delta^{2}\right)+\phi \delta\right]\right\}}{\lambda} .
$$

It follows from the previous derivative that:

$$
\frac{\partial \chi_{1}}{\partial \sigma_{\lambda}^{2}}>0 \Rightarrow-\left(1+\delta^{2}\right) \delta \phi(\bar{\lambda}-\lambda) \rho_{\pi}-\lambda\left(1+\delta^{2}-\beta \bar{\lambda} \rho_{\pi}\right) \phi \delta<\lambda\left(1+\delta^{2}-\beta \bar{\lambda} \rho_{\pi}\right)(\alpha-\gamma)\left(1+\delta^{2}\right) .
$$

Solving the above inequality for cases where $\bar{\lambda}<\frac{1+\delta^{2}}{\beta \rho_{\pi}}$ and $\bar{\lambda}>\frac{1+\delta^{2}}{\beta \rho_{\pi}}$ leads to the conditions given in Proposition 1d.

Q.E.D.

The first pair of conditions in Proposition 1d means that if $\bar{\lambda}$ is low enough so that $\bar{\lambda}<\frac{1+\delta^{2}}{\beta \rho_{\pi}}$, the effect of opacity on the real exchange rate persistence is positive if the demand of domestic goods depend more strongly on the real interest rate than on the real exchange rate in the sense that $\alpha-\gamma>-\frac{\delta \phi(\bar{\lambda}-\lambda) \rho_{\pi}}{\lambda\left[\left(1+\delta^{2}\right)-\beta \bar{\lambda} \rho_{\pi}\right]}-\frac{\phi \delta}{\left(1+\delta^{2}\right)}$. This condition becomes more restrictive if $\bar{\lambda}-\lambda<0$, i.e. the expected value of $\lambda$ is inferior to its realized value, and less restrictive if $\bar{\lambda}-\lambda<0$. Similar discussion can be made for the second pair of conditions.

\subsection{The effects of opacity and the persistence due to exchange rate shocks}

In order to analyze how opacity affects the pass-through of persistent exchange rate shocks to the inflation and real exchange rates, we derive the solutions of $\zeta_{2}, \chi_{2}, \mathrm{E}_{t} \zeta_{2}$ and $\mathrm{E}_{t} \chi_{3}$ given respectively by equations (29)-(31) with respect to the variance of $\lambda$. That gives the effect of opacity on the persistence of $\pi_{t}, e_{t}, \mathrm{E}_{t} \pi_{t+1}$, and $\mathrm{E}_{t} e_{t+1}$ due to persistent exchange rate shocks. The results are summarized in the following propositions.

Proposition 2a: An increase in opacity reinforces the effects of persistent exchange rate shocks on the expected future inflation when $\alpha-\gamma>0$ or $\alpha-\gamma<-\frac{\phi \delta}{1+\delta^{2}}$ but weakens them if $-\frac{\phi \delta}{1+\delta^{2}}<\alpha-\gamma<0$. 
Proof: In the case of exchange rate shocks, the effects of opacity on the expected future inflation is defined as $\frac{\partial \mathrm{E}_{t} \pi_{t+1}}{\partial \sigma_{\lambda}^{2}}=\frac{\rho_{e} \partial \mathrm{E}_{t} \zeta_{2}}{\partial \sigma_{\lambda}^{2}}$. Deriving $\mathrm{E}_{t} \zeta_{2}$ given by equation (31) with respect to $\sigma_{\lambda}^{2}$ gives:

$$
\frac{\partial \mathrm{E}_{t} \zeta_{2}}{\partial \sigma_{\lambda}^{2}}=\alpha \delta(\alpha-\gamma)\left(1+\delta^{2}\right)\left[(\alpha-\gamma)\left(1+\delta^{2}\right)+\phi \delta\right] \Omega^{2} \rho_{e}^{2} \phi^{2} \bar{\lambda}^{3} .
$$

It is straightforward to see that: $\frac{\partial \mathrm{E}_{t} \zeta_{2}}{\partial \sigma_{\lambda}^{2}}>0$ if $\alpha-\gamma>0$ or $\alpha-\gamma<-\frac{\phi \delta}{1+\delta^{2}}$; and $\frac{\partial \mathrm{E}_{t} \zeta_{2}}{\partial \sigma_{\lambda}^{2}}<0$ if $-\frac{\phi \delta}{1+\delta^{2}}<\alpha-\gamma<0$

Q.E.D.

In the presence of persistent exchange rate shocks, more opacity does not systematically induce an increase in the expected inflation and its effect depends on the respective but contradictory influence of the parameters $\gamma, \alpha, \phi$ and $\delta$. The direct transmission channel of exchange rate shocks through the goods market (IS curve) is neutralised by the optimal interest rate rule. Consequently, the opacity affects how persistent exchange rate shocks are transmitted to the expected future inflation first through the Phillips curve and then the IS curve. As we have discussed before, if the Phillips curve is independent of the real exchange rate, i.e. $\phi=0$, the expected future inflation will be determined uniquely by equation (16) and will only depend on inflation shock. Consequently, the opacity will have in this case no effect on the current and expected future inflation in the presence of persistent exchange rate shock.

A positive value to $\phi$ establishes the linkage between the exchange rate shocks and the current and expected future inflation through the Phillips curve. An increase in the value of $\phi$ implies that the inflation rate is more directly affected by exchange rate shocks. The higher is the value of $\phi$, the lower is the gain of competitiveness due to positive exchange rate shocks and hence more quickly the exchange rate shocks is transmitted to the current inflation as well as to inflation expectations due to their persistence.

In the presence of persistent exchange rate shocks, the effect of opacity is clearly tied with the introduction of real exchange rate effect in the Phillips curve, inducing complex interaction between endogenous variables through the Phillips curve, the IS curve and the UIP. If $\alpha-\gamma>0$, the effect of opacity on $\mathrm{E}_{t} \zeta_{2}$ is always positive since the effect of an increase in the expected future inflation dominates that due to an increase in the expected future real exchange rate on the 
domestic goods demand. For small values of $\alpha-\gamma$, the effect of opacity is also positive. It becomes negative for intermediate values of $\alpha-\gamma$.

Proposition 2b: i) An increase in opacity reinforces the effects of persistent exchange rate shocks on the expected future real exchange rate if one of the following pairs of conditions is satisfied:
1) $\bar{\lambda}<\frac{1+\delta^{2}}{\beta \rho_{e}}$ and $\alpha-\gamma>\max \left\{-\frac{\phi \delta}{1+\delta^{2}} ;-\frac{\phi \delta\left(1-\rho_{e}\right)}{1+\delta^{2}-\beta \bar{\lambda} \rho_{e}}\right\}$;
2) $\bar{\lambda}>\frac{1+\delta^{2}}{\beta \rho_{e}}$ and $-\frac{\phi \delta}{1+\delta^{2}}<\alpha-\gamma<-\frac{\phi \delta\left(1-\rho_{e}\right)}{1+\delta^{2}-\beta \bar{\lambda} \rho_{e}}$;
3) $\bar{\lambda}<\frac{1+\delta^{2}}{\beta \rho_{e}}$ and $\alpha-\gamma<\min \left\{-\frac{\phi \delta}{1+\delta^{2}} ;-\frac{\phi \delta\left(1-\rho_{e}\right)}{1+\delta^{2}-\beta \bar{\lambda} \rho_{e}}\right\}$.

ii) An increase in opacity reduces the effects of persistent exchange rate shocks on the expected future exchange rate if:
1) $\bar{\lambda}<\frac{1+\delta^{2}}{\beta}$ and $-\frac{\phi \delta}{1+\delta^{2}}<\alpha-\gamma<-\frac{\phi \delta\left(1-\rho_{e}\right)}{1+\delta^{2}-\beta \bar{\lambda} \rho_{e}}$;
2) $\bar{\lambda}>\frac{1+\delta^{2}}{\beta \rho_{e}}$ and $\alpha-\gamma>-\frac{\phi \delta\left(1-\rho_{e}\right)}{1+\delta^{2}-\beta \bar{\lambda} \rho_{e}}$;
3) $\frac{1+\delta^{2}}{\beta}<\bar{\lambda}<\frac{1+\delta^{2}}{\beta \rho_{e}}$ and $-\frac{\phi \delta\left(1-\rho_{e}\right)}{1+\delta^{2}-\beta \bar{\lambda} \rho_{e}}<\alpha-\gamma<-\frac{\phi \delta}{1+\delta^{2}}$;
4) $\bar{\lambda}>\frac{1+\delta^{2}}{\beta \rho_{e}}$ and $\alpha-\gamma<-\frac{\phi \delta}{1+\delta^{2}}$.

Proof: See Appendix C.

Q.E.D.

According to Proposition 2b, if $\bar{\lambda}$ is small enough, $\alpha-\gamma$ must be large enough or small enough to ensure that an increase in opacity reinforces the effect of persistent exchange rate shocks on the expected future exchange rate. In contrast, if $\bar{\lambda}$ is high enough, $\alpha-\gamma$ must be limited in an interval to produce the same effects. In these cases, more transparency allows reducing the transmission of persistent exchange rate shocks to the expected future real exchange rate. 
When $\bar{\lambda}$ is larger enough, an increase in opacity reduces the effects of persistent exchange rate shocks on the expected future exchange rate if $\alpha-\gamma$ is large enough or small enough. For intermediate values of $\alpha-\gamma$, more opacity can also reduce the effects of persistent exchange shock if $\bar{\lambda}<\frac{1+\delta^{2}}{\beta}$ or $\frac{1+\delta^{2}}{\beta}<\bar{\lambda}<\frac{1+\delta^{2}}{\beta \rho_{e}}$.

Proposition 2c: i) An increase in opacity reinforces the effects of persistent exchange rate shocks on the current inflation rate if one of the following conditions is checked:
1) $\alpha-\gamma>\max \left\{0 ;-\frac{\phi \delta}{1+\delta^{2}}\left(1-\frac{\lambda-\bar{\lambda}}{\lambda} \rho_{e}\right)\right\}$;
2) $\alpha-\gamma<\min \left\{0 ;-\frac{\phi \delta}{1+\delta^{2}}\left(1-\frac{\lambda-\bar{\lambda}}{\lambda} \rho_{e}\right)\right\}$.

ii) An increase in opacity reduces the effects of persistent exchange rate shocks on the current inflation rate if one of the following pairs of conditions is verified:

$$
\begin{aligned}
& \text { 1) } 0<\alpha-\gamma<-\frac{\phi \delta}{1+\delta^{2}}\left(1-\frac{\lambda-\bar{\lambda}}{\lambda} \rho_{e}\right) \text { and } \frac{\lambda-\bar{\lambda}}{\lambda}>\frac{1}{\rho_{e}} \\
& \text { 2) }-\frac{\phi \delta}{1+\delta^{2}}\left(1-\frac{\lambda-\bar{\lambda}}{\lambda} \rho_{e}\right)<\alpha-\gamma<0 \text { and } \frac{\lambda-\bar{\lambda}}{\lambda}<\frac{1}{\rho_{e}} \text {. }
\end{aligned}
$$

Proof: See Appendix D.

Q.E.D.

In Proposition 2c and similar to what has happened on the expected future inflation, if the value of $\alpha-\gamma$ is too high or too low, an increase in opacity reinforces the effect of persistent exchange rate shocks on the current inflation. In other words, more transparency helps diminishing the transmission of persistent exchange rate shocks to the current inflation. For intermediate values of $\alpha-\gamma$, an increase in opacity has the opposite effects. To the difference of the expected future inflation, the conditions defining the critical value of $\alpha-\gamma$ are limited by the rate of preference perception error $\left(\frac{\lambda-\bar{\lambda}}{\lambda}\right)$ when it concerns the current inflation rate.

We remark that in the conditions given in Proposition 2c, the expression $\gamma-\frac{\phi \delta}{1+\delta^{2}}\left(1-\frac{\lambda-\bar{\lambda}}{\lambda} \rho_{e}\right)$ is the similar to the one which appears in the condition in Proposition 1c. 
Proposition 2d: i) An increase in opacity reinforces the effects of persistent exchange rate shocks on the current exchange rate if either of the following groups of conditions is satisfied:
1) $\quad \bar{\lambda}<\frac{1+\delta^{2}}{\beta \rho_{e}}$ and $\alpha-\gamma>\max \left\{0 ;-\frac{\phi \delta}{1+\delta^{2}}+\frac{(\lambda-\bar{\lambda}) \delta \phi \rho_{e}}{\lambda\left(1+\delta^{2}-\beta \bar{\lambda} \rho_{e}\right)}\right\}$;
2) $\quad \bar{\lambda}<\frac{1+\delta^{2}}{\beta \rho_{e}}$ and $\alpha-\gamma<\min \left\{0 ;-\frac{\phi \delta}{1+\delta^{2}}+\frac{(\lambda-\bar{\lambda}) \delta \phi \rho_{e}}{\lambda\left(1+\delta^{2}-\beta \bar{\lambda} \rho_{e}\right)}\right\}$;
3) $\bar{\lambda}>\frac{1+\delta^{2}}{\beta \rho_{e}}$, $\frac{\lambda-\bar{\lambda}}{\lambda}<\frac{1+\delta^{2}-\beta \bar{\lambda} \rho_{e}}{\rho_{e}\left(1+\delta^{2}\right)}$ and $0<\alpha-\gamma<-\frac{\phi \delta}{1+\delta^{2}}+\frac{(\lambda-\bar{\lambda}) \delta \phi \rho_{e}}{\lambda\left(1+\delta^{2}-\beta \bar{\lambda} \rho_{e}\right)}$;
4) $\bar{\lambda}>\frac{1+\delta^{2}}{\beta \rho_{e}}, \frac{\lambda-\bar{\lambda}}{\lambda}>\frac{1+\delta^{2}-\beta \bar{\lambda} \rho_{e}}{\rho_{e}\left(1+\delta^{2}\right)}$ and $-\frac{\phi \delta}{1+\delta^{2}}+\frac{(\lambda-\bar{\lambda}) \delta \phi \rho_{e}}{\lambda\left(1+\delta^{2}-\beta \bar{\lambda} \rho_{e}\right)}<\alpha-\gamma<0$.

ii) An increase in opacity reduces the effects of persistent exchange rate shocks on the current real exchange rate if the first condition in the groups of conditions 1 and 2 is inversed, and the first and second conditions in the groups of conditions 3 and 4 are inversed.

Proof: See Appendix E. $\quad$ Q.E.D.

If $\bar{\lambda}<\frac{1+\delta^{2}}{\beta \rho_{e}}$, i.e. the central bank is perceived to be relatively conservative (with higher aversion to inflation), an increase in opacity reinforces the effects of persistent exchange rate shocks on the current real exchange rate both when $\alpha-\gamma$ is sufficiently large or small. When the central bank is perceived to be relatively accommodative, i.e. $\bar{\lambda}>\frac{1+\delta^{2}}{\beta \rho_{e}}$, an increase in opacity reinforces the effects of persistent exchange rate shocks for two intervals of intermediate values of $\alpha-\gamma$, which are positive if the rate of preference perception error is sufficiently small, i.e. $\frac{\lambda-\bar{\lambda}}{\lambda}<\frac{1+\delta^{2}-\beta \bar{\lambda} \rho_{e}}{\rho_{e}\left(1+\delta^{2}\right)}$, and negative in the opposite case. Similar comments can be made for the second part of Proposition 2d.

\section{The effects of opacity on macroeconomic volatility}

We examine in this section the effects of opacity on the variability of inflation and real exchange rate. Since the effect of opacity on the variability of output is similar to this on inflation, we will neglect it in the following. 
Using equations (17) and (18), the variances of inflation and real exchange rate are decomposed in three parts as follows:

$$
\begin{aligned}
& \operatorname{var}\left[\pi_{t}\right]=\zeta_{1}^{2} \operatorname{var}\left[\varepsilon_{t}^{\pi}\right]+\zeta_{2}^{2} \operatorname{var}\left[\varepsilon_{t}^{e}\right]+\zeta_{3}^{2} \operatorname{var}\left[\varepsilon_{t}^{x}\right], \\
& \operatorname{var}\left[e_{t}\right]=\chi_{1}^{2} \operatorname{var}\left[\varepsilon_{t}^{\pi}\right]+\chi_{2}^{2} \operatorname{var}\left[\varepsilon_{t}^{e}\right]+\chi_{3}^{2} \operatorname{var}\left[\varepsilon_{t}^{x}\right]
\end{aligned}
$$

Denote that $\operatorname{var}\left[\pi_{t}\right]=\sigma_{\pi}^{2}, \operatorname{var}\left[e_{t}\right]=\sigma_{e}^{2}, \operatorname{var}\left[\varepsilon_{t}^{\pi}\right]=\sigma_{\varepsilon^{\pi}}^{2}$ and $\operatorname{var}\left[\varepsilon_{t}^{e}\right]=\sigma_{\varepsilon^{e}}^{2}$. Using the value of $\zeta_{1}$ and $\chi_{1}$ given respectively by equation (25)-(26) in equations (33)-(34), we then derive the effect of opacity on the variances of inflation and real exchange rate due to persistent inflation shocks as follows:

$$
\begin{aligned}
& \frac{\partial^{2} \sigma_{\pi}^{2}}{\partial \sigma_{\varepsilon^{\pi}}^{2} \partial \sigma_{\lambda}^{2}}=2 \Psi^{3} \delta\left(1+\delta^{2}\right) \bar{\lambda}(\alpha-\gamma) \phi \rho_{\pi}^{2}\left\{\begin{array}{l}
(\bar{\lambda}-\lambda) \phi \delta(\alpha-\gamma) \bar{\lambda}^{3}\left(1-\rho_{\pi}\right) \rho_{\pi} \\
+\lambda(\alpha-\gamma) \bar{\lambda}^{3}\left(1-\rho_{\pi}\right)\left[(\alpha-\gamma)\left(1+\delta^{2}\right)+\phi \delta\right]
\end{array}\right\}^{2}, \\
& \frac{\partial^{2} \sigma_{e}^{2}}{\partial \sigma_{\varepsilon^{\pi}}^{2} \partial \sigma_{\lambda}^{2}}=2 \Psi^{3} \delta\left(1+\delta^{2}\right) \bar{\lambda}(\alpha-\gamma) \phi \rho_{\pi}^{2}\left(\frac{\delta \bar{\lambda}}{\lambda}\right)^{2}\left\{\begin{array}{l}
\bar{\lambda}^{2}\left[\left(1+\delta^{2}\right)(\alpha-\gamma)\left(\bar{\lambda} \rho_{\pi}-\lambda\right)-\lambda \delta \phi\left(1-\rho_{\pi}\right)\right] \\
+\left(1+\delta^{2}\right)(\alpha-\gamma)\left[\bar{\lambda}^{2}(\lambda-\bar{\lambda})+\lambda \sigma_{\lambda}^{2}\right] \rho_{\pi}^{2}
\end{array}\right\}^{2} .
\end{aligned}
$$

In the following, we derive conditions under which an increase in opacity reinforces the effects of persistent inflation and exchange rate shocks. In order to keep our paper at a reasonable length, we do not give the conditions under which an increase in opacity reduces the effects of persistent shocks on macroeconomic variability.

Proposition 3a: In the presence of persistent inflation shocks, an increase in opacity will increase the variance of both inflation and real exchange rate if $\Delta^{\pi}=\delta^{2} \phi^{2}\left[\Theta^{2}-4 \bar{\lambda}^{4}\left(1+\delta^{2}\right)\left(1+\delta^{2}-\beta \bar{\lambda} \rho_{\pi}\right)\left(1-\rho_{\pi}\right)^{2}\right]>0$ and one of the following groups of conditions is checked:

1) $\alpha-\gamma>\frac{\delta \phi \Theta+\sqrt{\Delta^{\pi}}}{2 \bar{\lambda}^{2}\left(1+\delta^{2}\right)\left(1+\delta^{2}-\beta \bar{\lambda} \rho_{\pi}\right)\left(1-\rho_{\pi}\right)}, \quad \bar{\lambda}<\frac{1+\delta^{2}}{\beta \rho_{\pi}}$ and $\Theta>0$;

2) $\alpha-\gamma>\frac{\delta \phi \Theta-\sqrt{\Delta^{\pi}}}{2 \bar{\lambda}^{2}\left(1+\delta^{2}\right)\left(1+\delta^{2}-\beta \bar{\lambda} \rho_{\pi}\right)\left(1-\rho_{\pi}\right)}, \bar{\lambda}>\frac{1+\delta^{2}}{\beta \rho_{\pi}}$ and $\Theta<0$;

3) $\alpha-\gamma<\frac{\delta \phi \Theta-\sqrt{\Delta^{\pi}}}{2 \bar{\lambda}^{2}\left(1+\delta^{2}\right)\left(1+\delta^{2}-\beta \bar{\lambda} \rho_{\pi}\right)\left(1-\rho_{\pi}\right)}, \quad \bar{\lambda}<\frac{1+\delta^{2}}{\beta \rho_{\pi}}$ and $\Theta>0$;

4) $\alpha-\gamma<\frac{\delta \phi \Theta+\sqrt{\Delta^{\pi}}}{2 \bar{\lambda}^{2}\left(1+\delta^{2}\right)\left(1+\delta^{2}-\beta \bar{\lambda} \rho_{\pi}\right)\left(1-\rho_{\pi}\right)}, \bar{\lambda}>\frac{1+\delta^{2}}{\beta \rho_{\pi}}$ and $\Theta<0$; 
5) $\frac{\delta \phi \Theta+\sqrt{\Delta^{\pi}}}{2 \bar{\lambda}^{2}\left(1+\delta^{2}\right)\left(1+\delta^{2}-\beta \bar{\lambda} \rho_{\pi}\right)\left(1-\rho_{\pi}\right)}<\alpha-\gamma<\frac{\delta \phi \Theta-\sqrt{\Delta^{\pi}}}{2 \bar{\lambda}^{2}\left(1+\delta^{2}\right)\left(1+\delta^{2}-\beta \bar{\lambda} \rho_{\pi}\right)\left(1-\rho_{\pi}\right)}, \bar{\lambda}>\frac{1+\delta^{2}}{\beta \rho_{\pi}}$ and $\Theta>0$;

6) $\frac{\delta \phi \Theta-\sqrt{\Delta^{\pi}}}{2 \bar{\lambda}^{2}\left(1+\delta^{2}\right)\left(1+\delta^{2}-\beta \bar{\lambda} \rho_{\pi}\right)\left(1-\rho_{\pi}\right)}<\alpha-\gamma<\frac{\delta \phi \Theta+\sqrt{\Delta^{\pi}}}{2 \bar{\lambda}^{2}\left(1+\delta^{2}\right)\left(1+\delta^{2}-\beta \bar{\lambda} \rho_{\pi}\right)\left(1-\rho_{\pi}\right)}, \bar{\lambda}<\frac{1+\delta^{2}}{\beta \rho_{\pi}}$ and $\Theta<0$;

where $\Theta=\beta \bar{\lambda}^{3}\left(1-\rho_{\pi}\right) \rho_{\pi}+\left(1+\delta^{2}\right)\left[\sigma_{\lambda}^{2} \rho_{\pi}^{2}-2 \bar{\lambda}^{2}\left(1-\rho_{\pi}\right)\right] . \quad \Theta>0 \quad(\Theta<0)$ is equivalent to $\sigma_{\lambda}^{2}>\max \left\{0, \frac{2 \bar{\lambda}^{2}\left(1-\rho_{\pi}\right)\left(1+\delta^{2}\right)-\beta \bar{\lambda}^{3}\left(1-\rho_{\pi}\right) \rho_{\pi}}{\rho_{\pi}^{2}\left(1+\delta^{2}\right)}\right\} \quad\left(\sigma_{\lambda}^{2}<\frac{2 \bar{\lambda}^{2}\left(1-\rho_{\pi}\right)\left(1+\delta^{2}\right)-\beta \bar{\lambda}^{3}\left(1-\rho_{\pi}\right) \rho_{\pi}}{\rho_{\pi}^{2}\left(1+\delta^{2}\right)}\right.$ respectively ).

Proof: See Appendix F.

Q.E.D.

According to equations (35)-(36), the persistent inflation shocks, and hence the opacity, have no effect on the variances of inflation and real exchange rate if their effects on the demand through two channels (the direct effect through the substitution effect due to a variation of real exchange rate and the indirect effect through the real interest rate) offset each other, i.e. $\alpha=\gamma$. In the case where $\alpha \neq \gamma$, an increase in opacity also has no effect on the variance of inflation if the rate of preference perception error is such that $\frac{\lambda-\bar{\lambda}}{\lambda}=\frac{(\alpha-\gamma)\left(1+\delta^{2}\right)+\phi \delta}{\phi \delta \rho_{\pi}}$. Similarly, an increase in opacity also has no effect on the variance of real exchange rate if the perception error of the public is such that $\frac{\lambda\left(1+\rho_{\pi}\right)-\bar{\lambda} \rho_{\pi}}{\lambda}=\frac{\left(1+\delta^{2}\right)(\alpha-\gamma) \rho_{\pi}^{2} \sigma_{\lambda}^{2}-\delta \phi\left(1-\rho_{\pi}\right) \bar{\lambda}^{2}}{\left(1-\rho_{\pi}\right)\left(1+\delta^{2}\right)(\alpha-\gamma) \bar{\lambda}^{2}}$.

In the cases where $\alpha \neq \gamma$ and where the conditions on the perception error leading to the absence of opacity effect are not satisfied, more opacity will increase the variance of inflation and that of real exchange rate both when $\alpha-\gamma$ is sufficiently large or small as shown by the conditions 1-4 of Proposition 3a, with restrictions imposed on the value of $\bar{\lambda}$ and the initial value of $\sigma_{\lambda}^{2}$. For intermediate values of $\alpha-\gamma$, more opacity will also increase the variance of inflation and that of real exchange rate under conditions 5 or 6 of Proposition 3a. Under the condition $5, \bar{\lambda}$ and $\sigma_{\lambda}^{2}$ must be sufficiently high as indicated by the associated supplementary conditions. In contrast, under condition 6 , the associated supplementary conditions show that $\bar{\lambda}$ and $\sigma_{\lambda}^{2}$ must be sufficiently low. 
The critical values for $\alpha-\gamma$, i.e. $\frac{\delta \phi \Theta \pm \sqrt{\Delta^{\pi}}}{2 \bar{\lambda}^{2}\left(1+\delta^{2}\right)\left(1+\delta^{2}-\beta \bar{\lambda} \rho_{\pi}\right)\left(1-\rho_{\pi}\right)(\alpha-\gamma)^{2}}$, which are important for determining the sense of the effect of opacity on the variances of inflation and real exchange rate, depend on other parameters. In particular, among these parameters, we remark that an increase in $\sigma_{\lambda}^{2}$ induces that of $\Theta$ and hence the critical value of $\alpha-\gamma$ and an increase in $\bar{\lambda}$ has ambiguous effects on it.

To determine how the opacity affects macroeconomic volatility in the presence of persistent exchange rate shocks, we use the solutions of $\zeta_{2}$ and $\chi_{2}$ given respectively by equation (29) and (30) in equations (33) and (34) and then we derive the effect of opacity on the variance of inflation and real exchange rate due to the persistent exchange rate shocks as follows:

$$
\begin{aligned}
& \frac{\partial^{2} \sigma_{\pi}^{2}}{\partial \sigma_{\varepsilon}^{2} e^{\partial \sigma_{\lambda}^{2}}}=2 \Omega^{3}\left(1+\delta^{2}\right)(\alpha-\gamma) \alpha^{2} \delta \phi^{3} \bar{\lambda}^{4} \rho_{e}^{2}\left\{(\bar{\lambda}-\lambda) \delta \phi \rho_{e}+\lambda\left[(\alpha-\gamma)\left(1+\delta^{2}\right)+\phi \delta\right]\right\}^{2}, \\
& \frac{\partial^{2} \sigma_{e}^{2}}{\partial \sigma_{\varepsilon}^{2} e^{\partial \sigma_{\lambda}^{2}}}=\frac{2 \Omega^{3}\left(1+\delta^{2}\right)(\alpha-\gamma) \alpha^{2} \delta \phi \bar{\lambda}^{4} \rho_{e}^{2}\left\{\left(1+\delta^{2}\right)(\bar{\lambda}-\lambda) \delta \phi \rho_{e}+\lambda\left[\left(1+\delta^{2}\right)-\beta \bar{\lambda} \rho_{e}\right]\left[(\alpha-\gamma)\left(1+\delta^{2}\right)+\phi \delta\right]\right\}^{2}}{\lambda^{2}} .
\end{aligned}
$$

Proposition 3b: In the presence of persistent exchange rate shocks, more opacity will increase the variance of inflation and that of real exchange rate if $\Delta^{e}=\left(1+\delta^{2}\right)^{2} \delta^{2} \phi^{2}\left[2 \bar{\lambda}^{2}\left(1-\rho_{e}\right)-\rho_{e}^{2} \sigma_{\lambda}^{2}\right]^{2}-4\left(1+\delta^{2}\right)\left(1-\rho_{e}\right)^{2}\left(1+\delta^{2}-\beta \bar{\lambda} \rho_{e}\right) \phi^{2} \delta^{2} \bar{\lambda}^{4}>0$ and if one of the following groups of conditions is checked:

1) $\alpha-\gamma>\frac{\left(1+\delta^{2}\right) \delta \phi\left[\rho_{e}^{2} \sigma_{\lambda}^{2}-2 \bar{\lambda}^{2}\left(1-\rho_{e}\right)\right]+\sqrt{\Delta^{e}}}{2\left(1+\delta^{2}\right)\left(1-\rho_{e}\right) \bar{\lambda}^{2}\left(1+\delta^{2}-\beta \bar{\lambda} \rho_{e}\right)}, \quad \bar{\lambda}<\frac{1+\delta^{2}}{\beta \rho_{e}}$ and $\sigma_{\lambda}^{2}>\frac{2 \bar{\lambda}^{2}\left(1-\rho_{e}\right)}{\rho_{e}^{2}}$

2) $\alpha-\gamma>\frac{\left(1+\delta^{2}\right) \delta \phi\left[\rho_{e}^{2} \sigma_{\lambda}^{2}-2 \bar{\lambda}^{2}\left(1-\rho_{e}\right)\right]-\sqrt{\Delta^{e}}}{2\left(1+\delta^{2}\right)\left(1-\rho_{e}\right) \bar{\lambda}^{2}\left(1+\delta^{2}-\beta \bar{\lambda} \rho_{e}\right)}, \bar{\lambda}>\frac{1+\delta^{2}}{\beta \rho_{e}}$ and $\sigma_{\lambda}^{2}<\frac{2 \bar{\lambda}^{2}\left(1-\rho_{e}\right)}{\rho_{e}^{2}}$;

3) $\alpha-\gamma<\frac{\left(1+\delta^{2}\right) \delta \phi\left[\rho_{e}^{2} \sigma_{\lambda}^{2}-2 \bar{\lambda}^{2}\left(1-\rho_{e}\right)\right]-\sqrt{\Delta^{e}}}{2\left(1+\delta^{2}\right)\left(1-\rho_{e}\right) \bar{\lambda}^{2}\left(1+\delta^{2}-\beta \bar{\lambda} \rho_{e}\right)}, \bar{\lambda}<\frac{1+\delta^{2}}{\beta \rho_{e}}$ and $\sigma_{\lambda}^{2}>\frac{2 \bar{\lambda}^{2}\left(1-\rho_{e}\right)}{\rho_{e}^{2}}$;

4) $\alpha-\gamma<\frac{\left(1+\delta^{2}\right) \delta \phi\left[\rho_{e}^{2} \sigma_{\lambda}^{2}-2 \bar{\lambda}^{2}\left(1-\rho_{e}\right)\right]+\sqrt{\Delta^{e}}}{2\left(1+\delta^{2}\right)\left(1-\rho_{e}\right) \bar{\lambda}^{2}\left(1+\delta^{2}-\beta \bar{\lambda} \rho_{e}\right)}, \bar{\lambda}>\frac{1+\delta^{2}}{\beta \rho_{e}}$ and $\sigma_{\lambda}^{2}<\frac{2 \bar{\lambda}^{2}\left(1-\rho_{e}\right)}{\rho_{e}^{2}}$;

5) $\quad \frac{\left(1+\delta^{2}\right) \delta \phi\left[\rho_{e}^{2} \sigma_{\lambda}^{2}-2 \bar{\lambda}^{2}\left(1-\rho_{e}\right)\right]+\sqrt{\Delta^{e}}}{2\left(1+\delta^{2}\right)\left(1-\rho_{e}\right) \bar{\lambda}^{2}\left(1+\delta^{2}-\beta \bar{\lambda} \rho_{e}\right)}<\alpha-\gamma<\frac{\left(1+\delta^{2}\right) \delta \phi\left[\rho_{e}^{2} \sigma_{\lambda}^{2}-2 \bar{\lambda}^{2}\left(1-\rho_{e}\right)\right]-\sqrt{\Delta^{e}}}{2\left(1+\delta^{2}\right)\left(1-\rho_{e}\right) \bar{\lambda}^{2}\left(1+\delta^{2}-\beta \bar{\lambda} \rho_{e}\right)}, \quad \bar{\lambda}>\frac{1+\delta^{2}}{\beta \rho_{e}} \quad$ and $\sigma_{\lambda}^{2}>\frac{2 \bar{\lambda}^{2}\left(1-\rho_{e}\right)}{\rho_{e}^{2}} ;$ 


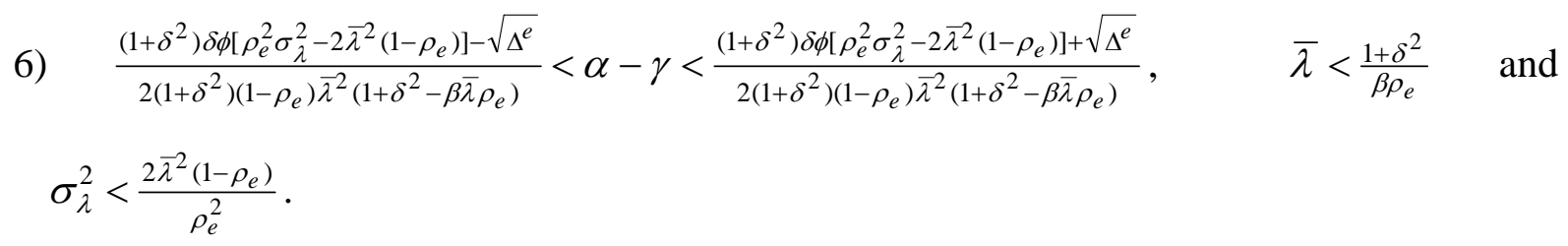

Proof: See Appendix G.

Q.E.D.

According to equations (37)-(38) and similar to persistent inflation shocks, persistent exchange rate shocks and hence the opacity have no effect on the variances of inflation and real exchange rate if their effects on the demand through the above mentioned two channels offset each other, i.e. $\alpha-\gamma=0$. In the case where $\alpha \neq \gamma$, an increase in opacity also has no effect on the variance of inflation if the rate of preference perception error is such that $\frac{\lambda-\bar{\lambda}}{\lambda}=\frac{(\alpha-\gamma)\left(1+\delta^{2}\right)+\phi \delta}{\delta \phi \rho_{e}}$. Similarly, an increase in opacity also has no effect on the variance of real exchange rate if the perception error is such that $\frac{\lambda-\bar{\lambda}}{\lambda}=\frac{\left[\left(1+\delta^{2}\right)-\beta \bar{\lambda} \rho_{e}\right]\left[(\alpha-\gamma)\left(1+\delta^{2}\right)+\phi \delta\right]}{\left(1+\delta^{2}\right) \delta \phi \rho_{e}}$.

In the cases where $\alpha \neq \gamma$ and the conditions on the preference perception error leading to the absence of opacity effect are not satisfied, the conditions under which an increase in opacity positively affects the variance of inflation and real exchange rate are quite similar to these defined in Proposition 3a, except the critical values for $\alpha-\gamma, \bar{\lambda}$ and $\sigma_{\lambda}^{2}$. Similar discussions can be made.

Having shown how opacity affects the level and volatility of economic variables, we can examine if there is a case for more opacity. When the central bank decided to increase the opacity about its preference, it accepted lower equilibrium inflation (and higher output gap) in exchange of greater macroeconomic instability. If the equilibrium level of inflation (and output gap) was increasing (and decreasing respectively) in opacity, there would be no such trade-off between the equilibrium level and volatility of inflation (and output gap) with respect to the degree of opacity. In the case where both inflation level and variability were increasing in opacity, the most desirable situation is that the central bank should be fully transparent $\left(\sigma_{\lambda}^{2}=0\right.$ ). Inversely, if both inflation and macroeconomic variability were decreasing in opacity, there would be a case for monetary policy opacity. 
According to Proposition 1c, we have $\frac{\partial \pi_{t}}{\partial \sigma_{\lambda}^{2}}<0$ if $\alpha-\gamma<-\frac{\phi \delta}{1+\delta^{2}}\left(1-\frac{\bar{\lambda}-\lambda}{\lambda} \rho_{\pi}\right)$. According to Proposition 3a, we have $\frac{\partial^{2} \sigma_{\pi}^{2}}{\partial \sigma_{\lambda}^{2}}>0$ under conditions 1-6. In the presence of persistent inflation shocks, the trade-off is possible if we have simultaneously $\frac{\partial \pi_{t}}{\partial \sigma_{\lambda}^{2}}<0$ and $\frac{\partial^{2} \sigma_{\pi}^{2}}{\partial \sigma_{\lambda}^{2}}>0$, i.e. the condition $\alpha-\gamma<-\frac{\phi \delta}{1+\delta^{2}}\left(1-\frac{\bar{\lambda}-\lambda}{\lambda} \rho_{\pi}\right)$ and one of the six groups of conditions in Proposition 3a are checked simultaneously. However, the case for transparency could be more compelling since Propositions 1c and 3a have shown that if $\alpha-\gamma$ is sufficiently large or low, more opacity could reinforces the effects of persistent inflation shocks on the level and volatility of inflation.

Similar discussions about the possibilities of trade-off between the levels of inflation and output gap and their volatility could be done in the presence of persistent exchange rate shocks. When there is no such trade-off, the cases for total transparency or total opacity could exist and the conditions could be examined in comparing the conditions under which we have simultaneously $\frac{\partial \pi_{t}}{\partial \sigma_{\lambda}^{2}}>0$ and $\frac{\partial^{2} \sigma_{\pi}^{2}}{\partial \sigma_{\lambda}^{2}}>0$ (or respectively $\frac{\partial \pi_{t}}{\partial \sigma_{\lambda}^{2}}<0$ and $\frac{\partial^{2} \sigma_{\pi}^{2}}{\partial \sigma_{\lambda}^{2}}<0$ ). We remark that, if the central bank had also the objective of stabilising the variability of real exchange rate, the conditions for a trade-off between inflation reduction and macroeconomic stability would be more complicated.

\section{Conclusion}

In this paper, we have analytically examined how the pass-through of persistent inflation and exchange rate shocks to the rest of the economy, in particular to the inflation and real exchange rate dynamics, is affected by the political transparency in monetary policymaking. Existent theoretical and empirical studies show that increasing central bank transparency could meaningfully diminish the volatility of inflation, real exchange rate and output gap. Furthermore, the adoption of flexible exchange rate regime could increase the incentive to be more transparent in the conduct of monetary policy.

If the effect of real exchange rate on the marginal cost is absent, the opacity affects the level and variability of endogenous variables only in the presence of persistent inflation shocks. When the marginal effect of real interest rate on the domestic goods demand is exactly compensated by 
that of real exchange rate, opacity has not any effect whatever is the nature of the shocks. Under some other conditions imposed on the perception error of the central bank preference, the opacity also could have no effect on macroeconomic variability.

The effect of opacity on the transmission of persistent inflation and exchange rate shocks to the levels of inflation and real exchange rate and their expected future values could be positive, null or negative, depending on the structural parameters of the economy as well as these characterizing the preferences of the central bank. More precisely, it depends on the interest elasticity of demand for domestic goods, the degree of trade openness, the effect of real exchange rate on the marginal cost and the slope of the Phillips curve, the perceived and realised weight that the central bank assigns to the output target, and the initial level of opacity. We have found that, generally if the difference between two key parameters, i.e. the interest elasticity of demand for domestic goods and the degree of trade openness, is sufficiently large or sufficiently low, an increase in opacity reinforces the effect of these shocks on the endogenous variables. For intermediate values of the difference between these two key parameters, an increase in opacity could reinforce or weaken the effect of persistent inflation and exchange rate shocks according to the conditions imposed on other structural parameters, including these characterising the central bank preferences.

We have also shown that an increase in opacity could increase the variance of inflation and real exchange rate both when the difference between the above-mentioned two key parameters is sufficiently large or low with critical values depending on all structural parameters characterising the economy as well as the central bank preferences (expected value of preference parameter, initial degree of opacity). For intermediate cases, more opacity could also increase the macroeconomic volatility under conditions imposed on the expected relative weight that the central bank assigns to output target and/or the initial degree of opacity.

Our result implies that when there is perfect capital mobility, high degree of domestic financial development, which is the case in many industrial countries, could justify an increase in the political transparency of monetary policymaking. This theoretical result coincides with some empirical evidences showing that countries adopting flexible exchange rate regime tend to be more transparent in the conduct of monetary policy.

\section{Appendix A: The case where $\phi=0$}


If $\phi=0$, the equation (16) becomes:

$$
E_{t} \pi_{t+1}=\frac{1+\delta^{2}}{\beta \lambda} \pi_{t}-\frac{1}{\beta} \varepsilon_{t}^{\pi}
$$

Using the method of undetermined coefficients to solve equaiton (A.1) and guessing that $\pi_{t}=\zeta_{1} \varepsilon_{t}^{\pi}$ and hence $E_{t} \pi_{t+1}=\rho_{\pi} \varepsilon_{t}^{\pi} \mathrm{E}_{t} \zeta_{1}$, we obtain:

$$
\mathrm{E}_{t} \zeta_{1} \rho_{\pi}=\frac{1+\delta^{2}}{\beta \lambda} \zeta_{1}-\frac{1}{\beta}
$$

Taking expectations of equation (A.2) and using second-order Taylor development, i.e. $\mathrm{E}_{t}\left[\frac{1+\delta^{2}}{\beta \lambda}\right]=\frac{1+\delta^{2}}{\beta \bar{\lambda}}+\frac{1+\delta^{2}}{\beta \bar{\lambda}^{3}} \sigma_{\lambda}^{2}+\ldots$, we obtain the solution of $\mathrm{E}_{t} \zeta_{1}$ and $\zeta_{1}$ as follows:

$$
\begin{aligned}
& \mathrm{E}_{t} \zeta_{1}=\frac{\bar{\lambda}^{3}}{\bar{\lambda}^{2}\left(1+\delta^{2}\right)+\left(1+\delta^{2}\right) \sigma_{\lambda}^{2}-\beta \bar{\lambda}^{3} \rho_{\pi}}, \\
& \zeta_{1}=\frac{\lambda\left[\bar{\lambda}^{2}\left(1+\delta^{2}\right)+\left(1+\delta^{2}\right) \sigma_{\lambda}^{2}\right]}{\left(1+\delta^{2}\right)\left[\bar{\lambda}^{2}\left(1+\delta^{2}\right)+\left(1+\delta^{2}\right) \sigma_{\lambda}^{2}-\beta \bar{\lambda}^{3} \rho_{\pi}\right]} .
\end{aligned}
$$

The effect of opacity is deduced from these solutions as follows:

$$
\begin{aligned}
& \frac{\partial \mathrm{E}_{t} \zeta_{1}}{\partial \sigma_{\lambda}^{2}}=\frac{-\bar{\lambda}^{3}\left(1+\delta^{2}\right)}{\left[\bar{\lambda}^{2}\left(1+\delta^{2}\right)+\left(1+\delta^{2}\right) \sigma_{\lambda}^{2}-\beta \bar{\lambda}^{3} \rho_{\pi}\right]^{2}}<0, \\
& \frac{\partial \zeta_{1}}{\partial \sigma_{\lambda}^{2}}=\frac{-\lambda\left(1+\delta^{2}\right) \beta \bar{\lambda}^{3} \rho_{\pi}}{\left(1+\delta^{2}\right)\left[\bar{\lambda}^{2}\left(1+\delta^{2}\right)+\left(1+\delta^{2}\right) \sigma_{\lambda}^{2}-\beta \bar{\lambda}^{3} \rho_{\pi}\right]^{2}}<0 .
\end{aligned}
$$

\section{Appendix B: Proof of Proposition $1 b$.}

The effects of opacity on $\mathrm{E}_{t} e_{t+1}$ can be obtained through the relationship $\frac{d \mathrm{E}_{t} e_{t+1}}{d \sigma_{\lambda}^{2}}=\frac{\rho_{\pi} d \mathrm{E}_{t} \chi_{1}}{d \sigma_{\lambda}^{2}}$. It is then sufficient to know the sign of $\frac{d \mathrm{E}_{t} \chi_{1}}{d \sigma_{\lambda}^{2}}$. Deriving $\mathrm{E}_{t} \chi_{1}$ given by equation (28) with respect to $\sigma_{\lambda}^{2}$ yields:

$$
\frac{d \mathrm{E}_{t} \chi_{1}}{d \sigma_{\lambda}^{2}}=-\Psi^{2} \delta\left(1+\delta^{2}\right) \bar{\lambda}^{2}(\alpha-\gamma) \rho_{\pi}\left(1-\rho_{\pi}\right)\left[\left(1+\delta^{2}\right)(\alpha-\gamma)+\phi \delta\right]\left[\beta \bar{\lambda}(\alpha-\gamma) \rho_{\pi}-(\alpha-\gamma)\left(1+\delta^{2}\right)-\phi \delta\left(1-\rho_{\pi}\right)\right] .
$$

i) An increase in opacity has positive effect on $\mathrm{E}_{t} \chi_{1}$ if the following condition is satisfied:

$$
(\alpha-\gamma)\left[\beta \bar{\lambda}(\alpha-\gamma) \rho_{\pi}-(\alpha-\gamma)\left(1+\delta^{2}\right)-\phi \delta\left(1-\rho_{\pi}\right)\right]\left[\left(1+\delta^{2}\right)(\alpha-\gamma)+\phi \delta\right]<0 .
$$

Four combinations of inequalities allowing the above condition to be checked are considered: 
The first combination, where we have simultaneously $(\alpha-\gamma)>0,\left(1+\delta^{2}\right)(\alpha-\gamma)+\phi \delta<0$ and $\beta \bar{\lambda}(\alpha-\gamma) \rho_{\pi}-(\alpha-\gamma)\left(1+\delta^{2}\right)-\phi \delta\left(1-\rho_{\pi}\right)>0$, has no solution since if $(\alpha-\gamma)>0$ then $\left[\left(1+\delta^{2}\right)(\alpha-\gamma)+\phi \delta\right]>0$.

The second combination, where we have concurrently $(\alpha-\gamma)>0,\left(1+\delta^{2}\right)(\alpha-\gamma)+\phi \delta>0$ and $\beta \bar{\lambda}(\alpha-\gamma) \rho_{\pi}-(\alpha-\gamma)\left(1+\delta^{2}\right)-\phi \delta\left(1-\rho_{\pi}\right)<0$, has one solution. The first two inequalities imply $\alpha-\gamma>0$. The third inequality is verified in two cases: if $\bar{\lambda}>\frac{1+\delta^{2}}{\beta \rho_{\pi}}$, we have $\alpha-\gamma>\frac{\phi \delta\left(1-\rho_{\pi}\right)}{\beta \bar{\lambda} \rho_{\pi}-1-\delta^{2}}$; if $\bar{\lambda}<\frac{1+\delta^{2}}{\beta \rho_{\pi}} \quad$ we have $\alpha-\gamma<\frac{\phi \delta\left(1-\rho_{\pi}\right)}{\beta \bar{\lambda} \rho_{\pi}-1-\delta^{2}}<0$. The final solution is: $\alpha-\gamma>\max \left\{0 ; \frac{\phi \delta\left(1-\rho_{\pi}\right)}{\beta \bar{\lambda} \rho_{\pi}-1-\delta^{2}}\right\}$ with $\bar{\lambda}>\frac{1+\delta^{2}}{\beta \rho_{\pi}}$.

The third combination, where we have simultaneously $(\alpha-\gamma)<0,\left(1+\delta^{2}\right)(\alpha-\gamma)+\phi \delta<0$ and $\beta \bar{\lambda}(\alpha-\gamma) \rho_{\pi}-(\alpha-\gamma)\left(1+\delta^{2}\right)-\phi \delta\left(1-\rho_{\pi}\right)<0$, has two solutions. Solving the first two inequalities yields: $\alpha-\gamma<-\frac{\phi \delta}{1+\delta^{2}}$. Solving the third inequality gives: if $\bar{\lambda}>\frac{1+\delta^{2}}{\beta \rho_{\pi}}$ and $\alpha-\gamma<\frac{\phi \delta\left(1-\rho_{\pi}\right)}{\beta \bar{\lambda} \rho_{\pi}-1-\delta^{2}}$; if $\bar{\lambda}<\frac{1+\delta^{2}}{\beta \rho_{\pi}}$ and $\alpha-\gamma>\frac{\phi \delta\left(1-\rho_{\pi}\right)}{\beta \bar{\lambda} \rho_{\pi}-1-\delta^{2}}$. Combing these conditions leads to: 1) $\alpha-\gamma<-\frac{\phi \delta}{1+\delta^{2}}$ if $\left.\bar{\lambda}>\frac{1+\delta^{2}}{\beta \rho_{\pi}} ; 2\right) \frac{\phi \delta\left(1-\rho_{\pi}\right)}{\beta \bar{\lambda} \rho_{\pi}-1-\delta^{2}}<\alpha-\gamma<-\frac{\phi \delta}{1+\delta^{2}}$ if $\bar{\lambda}<\frac{1+\delta^{2}}{\beta \rho_{\pi}}$.

The last combination, where we have simultaneously $(\alpha-\gamma)<0,\left(1+\delta^{2}\right)(\alpha-\gamma)+\phi \delta>0$ and $\beta \bar{\lambda}(\alpha-\gamma) \rho_{\pi}-(\alpha-\gamma)\left(1+\delta^{2}\right)-\phi \delta\left(1-\rho_{\pi}\right)>0$, has one solution. The first two inequalities imply: $-\frac{\phi \delta}{1+\delta^{2}}<\alpha-\gamma<0$. The third inequality yields: if $\bar{\lambda}>\frac{1+\delta^{2}}{\beta \rho_{\pi}}$, then $\alpha-\gamma>\frac{\phi \delta\left(1-\rho_{\pi}\right)}{\beta \bar{\lambda} \rho_{\pi}-1-\delta^{2}}>0$; if $\bar{\lambda}<\frac{1+\delta^{2}}{\beta \rho_{\pi}}$ then $\alpha-\gamma<\frac{\phi \delta\left(1-\rho_{\pi}\right)}{\beta \bar{\lambda} \rho_{\pi}-1-\delta^{2}}<0$. That leads to: $\bar{\lambda}<\frac{1+\delta^{2}}{\beta \rho_{\pi}}$ and $-\frac{\phi \delta}{1+\delta^{2}}<\alpha-\gamma<\frac{\phi \delta\left(1-\rho_{\pi}\right)}{\beta \bar{\lambda} \rho_{\pi}-1-\delta^{2}}$.

ii) An increase in opacity has negative effect on $E_{t} \chi_{1}$ if the following condition is satisfied:

$$
(\alpha-\gamma)\left[\beta \bar{\lambda}(\alpha-\gamma) \rho_{\pi}-(\alpha-\gamma)\left(1+\delta^{2}\right)-\phi \delta\left(1-\rho_{\pi}\right)\right]\left[\left(1+\delta^{2}\right)(\alpha-\gamma)+\phi \delta\right]>0 .
$$

Considering similarly four combinations of inequalities as before and solving them, we obtain the pairs of conditions given in the second part of Proposition $1 \mathrm{~b}$.

Q.E.D.

\section{Appendix C: Proof of Proposition 2b}


In the presence of persistent exchange rate shocks, the effect of opacity on $\mathrm{E}_{t} e_{t+1}$ $\left(\frac{\partial E e_{t+1}}{\partial \sigma_{\lambda}^{2}}=\frac{\rho_{e} \partial \chi_{2}}{\partial \sigma_{\lambda}^{2}}\right)$ is obtained by deriving $\mathrm{E}_{t} \chi_{2}$ given by equation (32) with respect to $\sigma_{\lambda}^{2}$ as follows:

$$
\begin{aligned}
\frac{\partial \mathrm{E}_{t} \chi_{2}}{\partial \sigma_{\lambda}^{2}}= & \delta\left(1+\delta^{2}\right) \phi(\alpha-\gamma) \rho_{e}^{2} \Omega^{2}\left\{\left[\left(1+\delta^{2}\right)(\alpha-\gamma)+\phi \delta\right]\left[\left(1+\delta^{2}\right)-\bar{\lambda} \beta \rho_{e}\right] \alpha \bar{\lambda}^{2}+\left(1+\delta^{2}\right) \phi \alpha \delta \rho_{e} \sigma_{\lambda}^{2}\right\} \\
& +\Omega\left(1+\delta^{2}\right) \phi \alpha \delta \rho_{e} .
\end{aligned}
$$

i) Using the definition of $\Omega$ in the above derivative and after some rearrangement, we find that having $\frac{\partial \chi_{2}}{\partial \sigma_{\lambda}^{2}}>0$ is equivalent to have:

$$
\Omega^{2}\left[\left(1+\delta^{2}\right)(\alpha-\gamma)+\phi \delta\right]\left\{\left(1+\delta^{2}\right)(\alpha-\gamma)+\phi \delta-[\beta \bar{\lambda}(\alpha-\gamma)+\phi \delta] \rho_{e}\right\} \alpha \bar{\lambda}^{2}>0 .
$$

The above inequality is true in two cases.

In the first case, we must have simultaneously $\left(1+\delta^{2}\right)(\alpha-\gamma)+\phi \delta>0$ and $\left(1+\delta^{2}\right)(\alpha-\gamma)+\phi \delta-[\beta \bar{\lambda}(\alpha-\gamma)+\phi \delta] \rho_{e}>0$. That yields two solutions: 1) If $\bar{\lambda}<\frac{1+\delta^{2}}{\beta \rho_{e}}$, these two inequalities imply that $\alpha-\gamma>-\frac{\phi \delta}{1+\delta^{2}}$ and $(\alpha-\gamma)>-\frac{\phi \delta\left(1-\rho_{e}\right)}{\left(1+\delta^{2}-\beta \bar{\lambda} \rho_{e}\right)}$, that leads to $(\alpha-\gamma)>\max \left\{-\frac{\phi \delta}{1+\delta^{2}} ;-\frac{\phi \delta\left(1-\rho_{e}\right)}{1+\delta^{2}-\beta \bar{\lambda} \rho_{e}}\right\}$. 2) If $\bar{\lambda}>\frac{1+\delta^{2}}{\beta \rho_{e}}$, the solution satisfying these two inequalities is $-\frac{\phi \delta}{1+\delta^{2}}<\alpha-\gamma<-\frac{\phi \delta\left(1-\rho_{e}\right)}{1+\delta^{2}-\beta \bar{\lambda} \rho_{e}}$.

In the second case, we must have simultaneously $\left(1+\delta^{2}\right)(\alpha-\gamma)+\phi \delta<0$ and $\left(1+\delta^{2}\right)(\alpha-\gamma)+\phi \delta-[\beta \bar{\lambda}(\alpha-\gamma)+\phi \delta] \rho_{e}<0$. That leads to another two possible solutions: 1$)$ If $\bar{\lambda}<\frac{1+\delta^{2}}{\beta \rho_{e}}$, the solution satisfying these two equalities is $\left.\alpha-\gamma<\min \left\{-\frac{\phi \delta}{1+\delta^{2}} ;-\frac{\phi \delta\left(1-\rho_{e}\right)}{1+\delta^{2}-\beta \bar{\lambda} \rho_{e}}\right\} .2\right)$ If $\bar{\lambda}>\frac{1+\delta^{2}}{\beta \rho_{e}}$, we must have simultaneously $\alpha-\gamma<-\frac{\phi \delta}{1+\delta^{2}}$ and $\alpha-\gamma>-\frac{\phi \delta\left(1-\rho_{e}\right)}{1+\delta^{2}-\beta \bar{\lambda} \rho_{e}}$. Since $\bar{\lambda}>\frac{1+\delta^{2}}{\beta \rho_{e}}$ implies $1+\delta^{2}-\beta \bar{\lambda} \rho_{e}<0$, the second solution cannot be viable and only the first solution is valid.

ii) Consider the conditions under which we have $\frac{\partial \chi_{2}}{\partial \sigma_{\lambda}^{2}}<0$. The latter is equivalent to have:

$$
\Omega^{2}\left[\left(1+\delta^{2}\right)(\alpha-\gamma)+\phi \delta\right]\left\{\left(1+\delta^{2}\right)(\alpha-\gamma)+\phi \delta-[\beta \bar{\lambda}(\alpha-\gamma)+\phi \delta] \rho_{e}\right\} \alpha \bar{\lambda}^{2}<0
$$


In the first case, we must have simultaneously $\left(1+\delta^{2}\right)(\alpha-\gamma)+\phi \delta>0$ and $\left(1+\delta^{2}\right)(\alpha-\gamma)+\phi \delta-[\beta \bar{\lambda}(\alpha-\gamma)+\phi \delta] \rho_{e}<0$. That yields two solutions: 1$)$ If $\bar{\lambda}<\frac{1+\delta^{2}}{\beta \rho_{e}}$, we have $-\frac{\phi \delta}{1+\delta^{2}}<\alpha-\gamma<-\frac{\phi \delta\left(1-\rho_{e}\right)}{1+\delta^{2}-\beta \bar{\lambda} \rho_{e}}$. For the latter condition to be checked, we must have $\bar{\lambda}<\frac{1+\delta^{2}}{\beta}$, which is compatible with the initial condition $\bar{\lambda}<\frac{1+\delta^{2}}{\beta \rho_{e}}$. The latter condition implies $\bar{\lambda}<\frac{1+\delta^{2}}{\beta}$. 2) If $\bar{\lambda}>\frac{1+\delta^{2}}{\beta \rho_{e}}$, we must have simultaneously $\alpha-\gamma>-\frac{\phi \delta}{1+\delta^{2}}$ and $\alpha-\gamma>-\frac{\phi \delta\left(1-\rho_{e}\right)}{1+\delta^{2}-\beta \bar{\lambda} \rho_{e}}$, as the condition $\bar{\lambda}>\frac{1+\delta^{2}}{\beta \rho_{e}}$ implies that $-\frac{\phi \delta\left(1-\rho_{e}\right)}{1+\delta^{2}-\beta \bar{\lambda} \rho_{e}}>0$, then the solution is $\alpha-\gamma>-\frac{\phi \delta\left(1-\rho_{e}\right)}{1+\delta^{2}-\beta \bar{\lambda} \rho_{e}}$.

In the second case, we must have simultaneously $\left(1+\delta^{2}\right)(\alpha-\gamma)+\phi \delta<0$ and $\left(1+\delta^{2}\right)(\alpha-\gamma)+\phi \delta-[\beta \bar{\lambda}(\alpha-\gamma)+\phi \delta] \rho_{e}>0$. That leads to another two possible solutions: 1$)$ If $\bar{\lambda}<\frac{1+\delta^{2}}{\beta \rho_{e}}$, we obtain $-\frac{\phi \delta\left(1-\rho_{e}\right)}{1+\delta^{2}-\beta \bar{\lambda} \rho_{e}}<\alpha-\gamma<-\frac{\phi \delta}{1+\delta^{2}}$. The latter condition implies $\frac{1+\delta^{2}}{\beta}<\bar{\lambda}$, which is consistent with $\bar{\lambda}<\frac{1+\delta^{2}}{\beta \rho_{e}} \quad$ 2) If $\bar{\lambda}>\frac{1+\delta^{2}}{\beta \rho_{e}}$, we must have simultaneously $\alpha-\gamma<-\frac{\phi \delta}{1+\delta^{2}}$ $\alpha-\gamma<-\frac{\phi \delta\left(1-\rho_{e}\right)}{1+\delta^{2}-\beta \bar{\lambda} \rho_{e}}$. Since $-\frac{\phi \delta\left(1-\rho_{e}\right)}{1+\delta^{2}-\beta \bar{\lambda} \rho_{e}}>0$, it yields that $\alpha-\gamma<-\frac{\phi \delta}{1+\delta^{2}}$.

Q.E.D.

\section{Appendix D: Proof of Proposition 2c}

In the presence of persistent exchange rate shocks, the effects of opacity on inflation persistence ( $\frac{\partial \pi_{t}}{\partial \sigma_{\lambda}^{2}}=\frac{\rho_{e} \partial \zeta_{2}}{\partial \sigma_{\lambda}^{2}}$ ) is obtained by deriving $\zeta_{2}$ given by (29) with respect to $\sigma_{\lambda}^{2}$ :

$$
\frac{\partial \zeta_{2}}{\partial \sigma_{\lambda}^{2}}=\Omega^{2}\left(1+\delta^{2}\right)(\alpha-\gamma) \delta \phi \rho_{e} \rho_{e} \alpha \phi \bar{\lambda}^{2}\left\{(\bar{\lambda}-\lambda) \delta \phi \rho_{e}+\lambda\left[(\alpha-\gamma)\left(1+\delta^{2}\right)+\phi \delta\right]\right\}
$$

We have $\frac{\partial \zeta_{2}}{\partial \sigma_{\lambda}^{2}}>0$ in two cases: In the first case, we must have simultaneously $\alpha-\gamma>0$ and $(\bar{\lambda}-\lambda) \delta \phi \rho_{e}+\lambda\left[(\alpha-\gamma)\left(1+\delta^{2}\right)+\phi \delta\right]>0$. That leads to:

$$
\alpha-\gamma>\max \left\{0 ;-\frac{\phi \delta}{1+\delta^{2}}\left(1-\frac{\lambda-\bar{\lambda}}{\lambda} \rho_{e}\right)\right\}
$$

In the second case, we must have simultaneously $\alpha-\gamma<0$ and $(\bar{\lambda}-\lambda) \delta \phi \rho_{e}+\lambda\left[(\alpha-\gamma)\left(1+\delta^{2}\right)+\phi \delta\right]<0$. That yields: 


$$
\alpha-\gamma<\min \left\{0 ;-\frac{\phi \delta}{1+\delta^{2}}\left(1-\frac{\lambda-\bar{\lambda}}{\lambda} \rho_{e}\right)\right\}
$$

To obtain negative effect of opacity, i.e. $\frac{\partial \zeta_{2}}{\partial \sigma_{\lambda}^{2}}<0$, it is equivalent to have $(\alpha-\gamma)\left\{(\bar{\lambda}-\lambda) \delta \phi \rho_{e}+\lambda\left[(\alpha-\gamma)\left(1+\delta^{2}\right)+\phi \delta\right]\right\}<0$. Two cases are distinguished. In the first case, we must have simultaneously $\alpha-\gamma>0$ and $(\bar{\lambda}-\lambda) \delta \phi \rho_{e}+\lambda\left[(\alpha-\gamma)\left(1+\delta^{2}\right)+\phi \delta\right]<0$. That leads to $0<\alpha-\gamma<-\frac{\phi \delta}{1+\delta^{2}}\left(1-\frac{\lambda-\bar{\lambda}}{\lambda} \rho_{e}\right)$. This condition is valid only if $\frac{\lambda-\bar{\lambda}}{\lambda}>\frac{1}{\rho_{e}}$.

In the second case, we must have simultaneously $\alpha-\gamma<0$ and $(\bar{\lambda}-\lambda) \delta \phi \rho_{e}+\lambda\left[(\alpha-\gamma)\left(1+\delta^{2}\right)+\phi \delta\right]>0 . \quad$ Solving these two inequalities leads to: $-\frac{\phi \delta}{1+\delta^{2}}\left(1-\frac{\lambda-\bar{\lambda}}{\lambda} \rho_{e}\right)<\alpha-\gamma<0$. This condition is valid only if $\frac{\lambda-\bar{\lambda}}{\lambda}<\frac{1}{\rho_{e}}$. Q.E.D.

\section{Appendix E: Proof of Proposition 2d}

In the presence of persistent exchange rate shocks, the effects of opacity on $e_{t}$ $\left(\frac{\partial \pi_{t}}{\partial \sigma_{\lambda}^{2}}=\frac{\rho_{e} \partial \chi_{2}}{\partial \sigma_{\lambda}^{2}}\right)$ is obtained by deriving $\chi_{2}$ given by equation (30) with respect to $\sigma_{\lambda}^{2}$ :

$$
\frac{\partial \chi_{2}}{\partial \sigma_{\lambda}^{2}}=\frac{\Omega^{2} \delta\left(1+\delta^{2}\right) \phi(\alpha-\gamma) \rho_{e}^{2} \alpha \bar{\lambda}^{2}}{\lambda}\left\{\left(1+\delta^{2}\right)(\bar{\lambda}-\lambda) \delta \phi \rho_{e}+\lambda\left[\left(1+\delta^{2}\right)-\beta \bar{\lambda} \rho_{e}\right]\left[(\alpha-\gamma)\left(1+\delta^{2}\right)+\phi \delta\right]\right\} .
$$

We obtain $\frac{\partial \chi_{2}}{\partial \sigma_{\lambda}^{2}}>0$ in two cases. In the first case, we must have simultaneously $\alpha-\gamma>0$ and $\left(1+\delta^{2}\right)(\bar{\lambda}-\lambda) \delta \phi \rho_{e}+\lambda\left(1+\delta^{2}-\beta \bar{\lambda} \rho_{e}\right)\left[(\alpha-\gamma)\left(1+\delta^{2}\right)+\phi \delta\right]>0$. That leads to two solutions: 1$)$ $\alpha-\gamma>\max \left\{0 ;-\frac{\phi \delta}{1+\delta^{2}}+\frac{(\lambda-\bar{\lambda}) \delta \phi \rho_{e}}{\lambda\left(1+\delta^{2}-\beta \bar{\lambda} \rho_{e}\right)}\right\} \quad$ if $\left.\quad \bar{\lambda}<\frac{1+\delta^{2}}{\beta \rho_{e}} ; \quad 2\right) \quad 0<\alpha-\gamma<-\frac{\phi \delta}{1+\delta^{2}}+\frac{(\lambda-\bar{\lambda}) \delta \phi \rho_{e}}{\lambda\left(1+\delta^{2}-\beta \bar{\lambda} \rho_{e}\right)} \quad$ if $\bar{\lambda}>\frac{1+\delta^{2}}{\beta \rho_{e}}$. The last solution is valid only when we have $\frac{\lambda-\bar{\lambda}}{\lambda}<\frac{1+\delta^{2}-\beta \bar{\lambda} \rho_{e}}{\rho_{e}\left(1+\delta^{2}\right)}$.

In the second case, we must have simultaneously $\alpha-\gamma<0$ and $\left(1+\delta^{2}\right)(\bar{\lambda}-\lambda) \delta \phi \rho_{e}+\lambda\left(1+\delta^{2}-\beta \bar{\lambda} \rho_{e}\right)\left[(\alpha-\gamma)\left(1+\delta^{2}\right)+\phi \delta\right]<0$. There are two solutions: 1$)$ $\alpha-\gamma<\min \left\{0 ;-\frac{\phi \delta}{1+\delta^{2}}+\frac{(\lambda-\bar{\lambda}) \delta \phi \rho_{e}}{\lambda\left(1+\delta^{2}-\beta \bar{\lambda} \rho_{e}\right)}\right\} \quad$ if $\left.\quad \bar{\lambda}<\frac{1+\delta^{2}}{\beta \rho_{e}} ; \quad 2\right) \quad-\frac{\phi \delta}{1+\delta^{2}}+\frac{(\lambda-\bar{\lambda}) \delta \phi \rho_{e}}{\lambda\left(1+\delta^{2}-\beta \bar{\lambda} \rho_{e}\right)}<\alpha-\gamma<0 \quad$ if $\bar{\lambda}>\frac{1+\delta^{2}}{\beta \rho_{e}}$. The last solution is valid only when we have $\frac{\lambda-\bar{\lambda}}{\lambda}>\frac{1+\delta^{2}-\beta \bar{\lambda} \rho_{e}}{\rho_{e}\left(1+\delta^{2}\right)}$. 
We can have $\frac{\partial \chi_{2}}{\partial \sigma_{\lambda}^{2}}<0$ in two cases. In the first case, we must have simultaneously $\alpha-\gamma>0$ and $\left(1+\delta^{2}\right)(\bar{\lambda}-\lambda) \delta \phi \rho_{e}+\lambda\left(1+\delta^{2}-\beta \bar{\lambda} \rho_{e}\right)\left[(\alpha-\gamma)\left(1+\delta^{2}\right)+\phi \delta\right]<0$. There are two solutions: 1$)$ If $\bar{\lambda}<\frac{1+\delta^{2}}{\beta \rho_{e}}$, then $0<\alpha-\gamma<-\frac{\phi \delta}{1+\delta^{2}}+\frac{(\lambda-\bar{\lambda}) \delta \phi \rho_{e}}{\lambda\left(1+\delta^{2}-\beta \bar{\lambda} \rho_{e}\right)}$. The last condition is valid only when we have $\left.\frac{\lambda-\bar{\lambda}}{\lambda}>\frac{1+\delta^{2}-\beta \bar{\lambda} \rho_{e}}{\rho_{e}\left(1+\delta^{2}\right)} ; 2\right)$ If $\bar{\lambda}>\frac{1+\delta^{2}}{\beta \rho_{e}}$, then we have: $\alpha-\gamma>\max \left\{0 ;-\frac{\phi \delta}{1+\delta^{2}}+\frac{(\lambda-\bar{\lambda}) \delta \phi \rho_{e}}{\lambda\left(1+\delta^{2}-\beta \bar{\lambda} \rho_{e}\right)}\right\}$.

In the second case, we must have simultaneously $\alpha-\gamma<0$ and $\left(1+\delta^{2}\right)(\bar{\lambda}-\lambda) \delta \phi \rho_{e}+\lambda\left(1+\delta^{2}-\beta \bar{\lambda} \rho_{e}\right)\left[(\alpha-\gamma)\left(1+\delta^{2}\right)+\phi \delta\right]>0$. There are two solutions: 1$)$ $-\frac{\phi \delta}{1+\delta^{2}}+\frac{(\lambda-\bar{\lambda}) \delta \phi \rho_{e}}{\lambda\left(1+\delta^{2}-\beta \bar{\lambda} \rho_{e}\right)}<\alpha-\gamma<0 \quad$ if $\quad \bar{\lambda}<\frac{1+\delta^{2}}{\beta \rho_{e}} \quad$ and $\quad \frac{\lambda-\bar{\lambda}}{\lambda}<\frac{1+\delta^{2}-\beta \bar{\lambda} \rho_{e}}{\rho_{e}\left(1+\delta^{2}\right)}$ $\alpha-\gamma<\min \left\{0 ;-\frac{\phi \delta}{1+\delta^{2}}+\frac{(\lambda-\bar{\lambda}) \delta \phi \rho_{e}}{\lambda\left(1+\delta^{2}-\beta \bar{\lambda} \rho_{e}\right)}\right\}$ if $\bar{\lambda}>\frac{1+\delta^{2}}{\beta \rho_{e}} \quad$ Q.E.D.

\section{Appendix F: Proof of Proposition 3a}

According to equations (35) and (36), if $\alpha \neq \gamma, \frac{\lambda-\bar{\lambda}}{\lambda} \neq \frac{(\alpha-\gamma)\left(1+\delta^{2}\right)+\phi \delta}{\phi \delta \rho_{\pi}}$ and $\frac{\lambda\left(1+\rho_{\pi}\right)-\bar{\lambda} \rho_{\pi}}{\lambda} \neq \frac{\left(1+\delta^{2}\right)(\alpha-\gamma) \rho_{\pi}^{2} \sigma_{\lambda}^{2}-\delta \phi\left(1-\rho_{\pi}\right) \bar{\lambda}^{2}}{\left(1-\rho_{\pi}\right)\left(1+\delta^{2}\right)(\alpha-\gamma) \bar{\lambda}^{2}}$, the sign of $\frac{\partial^{2} \sigma_{\pi}^{2}}{\partial \sigma_{\varepsilon}^{2} \partial \sigma_{\lambda}^{2}}$ as well as $\frac{\partial^{2} \sigma_{e}^{2}}{\partial \sigma_{\varepsilon}^{2} \partial \sigma_{\lambda}^{2}}$ is the same as that of $\Psi(\alpha-\gamma)$. Hence, we only study the conditions under which $\Psi(\alpha-\gamma)$ is positive. To have $\Psi(\alpha-\gamma)>0$ is equivalent to have one of the following groups of conditions satisfied:

1) $\Psi>0$ and $\alpha-\gamma>0$;

2) $\Psi<0$ and $\alpha-\gamma<0$.

Taking account of the definition of $\Psi$, it is easy to show that the inequality $\Psi>0$ is equivalent to

$-\left\{\delta\left[\left(1+\delta^{2}\right)-\bar{\lambda} \beta\right] \bar{\lambda}^{2}+\delta\left(1+\delta^{2}\right) \sigma_{\lambda}^{2}\right\} \bar{\lambda}(\alpha-\gamma) \phi \rho_{\pi}^{2}+$ $\bar{\lambda}^{3}\left\{\left(1+\delta^{2}\right)(\alpha-\gamma) \rho_{\pi}-\left[(\alpha-\gamma)\left(1+\delta^{2}\right)+\phi \delta\right]\right\}\left\{[\beta \bar{\lambda}(\alpha-\gamma)+\phi \delta] \rho_{\pi}-(\alpha-\gamma)\left(1+\delta^{2}\right)-\phi \delta\right\}>0$,

which after some arrangements and simplification becomes:

$$
\bar{\lambda}^{2}\left(1+\delta^{2}\right)\left(1+\delta^{2}-\beta \bar{\lambda} \rho_{\pi}\right)\left(1-\rho_{\pi}\right)(\alpha-\gamma)^{2}-\delta \phi \Theta(\alpha-\gamma)+\left(1-\rho_{\pi}\right) \bar{\lambda}^{2} \phi^{2} \delta^{2}>0,
$$

or equivalently: 


$$
\left\{\alpha-\gamma-\frac{\delta \phi \Theta+\sqrt{\Delta^{\pi}}}{2 \bar{\lambda}^{2}\left(1+\delta^{2}\right)\left(1+\delta^{2}-\beta \bar{\lambda} \rho_{\pi}\right)\left(1-\rho_{\pi}\right)}\right\}\left\{\alpha-\gamma-\frac{\delta \phi \Theta-\sqrt{\Delta^{\pi}}}{2 \bar{\lambda}^{2}\left(1+\delta^{2}\right)\left(1+\delta^{2}-\beta \bar{\lambda} \rho_{\pi}\right)\left(1-\rho_{\pi}\right)}\right\}>0,
$$

where $\Theta=\beta \bar{\lambda}^{3}\left(1-\rho_{\pi}\right) \rho_{\pi}+\left(1+\delta^{2}\right) \sigma_{\lambda}^{2} \rho_{\pi}^{2}-2\left(1+\delta^{2}\right) \bar{\lambda}^{2}\left(1-\rho_{\pi}\right)$.

If $\Delta^{\pi}=(\delta \phi \Theta)^{2}-4\left(1+\delta^{2}\right)\left(1+\delta^{2}-\beta \bar{\lambda} \rho_{\pi}\right)\left(1-\rho_{\pi}\right)^{2} \delta^{2} \phi^{2} \bar{\lambda}^{4}>0$, we can solve the above inequality in distinguishing two cases as follows.

In the first case, both expressions in braces must be positive, i.e. $\alpha-\gamma-\frac{\delta \phi \Theta \pm \sqrt{\Delta^{\pi}}}{2 \bar{\lambda}^{2}\left(1+\delta^{2}\right)\left(1+\delta^{2}-\beta \bar{\lambda} \rho_{\pi}\right)\left(1-\rho_{\pi}\right)}>0$. That leads to two solutions:

1) $\alpha-\gamma>\frac{\delta \phi \ominus+\sqrt{\Delta^{\pi}}}{2 \bar{\lambda}^{2}\left(1+\delta^{2}\right)\left(1+\delta^{2}-\beta \bar{\lambda} \rho_{\pi}\right)\left(1-\rho_{\pi}\right)}$, if $\bar{\lambda}<\frac{1+\delta^{2}}{\beta \rho_{\pi}}$;

2) $\alpha-\gamma>\frac{\delta \phi \Theta-\sqrt{\Delta^{\pi}}}{2 \bar{\lambda}^{2}\left(1+\delta^{2}\right)\left(1+\delta^{2}-\beta \bar{\lambda} \rho_{\pi}\right)\left(1-\rho_{\pi}\right)}>0$, if $\bar{\lambda}>\frac{1+\delta^{2}}{\beta \rho_{\pi}}$.

In the second case, both expressions in braces must be negative, i.e. $\alpha-\gamma-\frac{\delta \phi \boxminus \pm \sqrt{\Delta^{\pi}}}{2 \bar{\lambda}^{2}\left(1+\delta^{2}\right)\left(1+\delta^{2}-\beta \bar{\lambda} \rho_{\pi}\right)\left(1-\rho_{\pi}\right)}<0$. That yields another two solutions:

1) $\alpha-\gamma<\frac{\delta \phi \Theta-\sqrt{\Delta^{\pi}}}{2 \bar{\lambda}^{2}\left(1+\delta^{2}\right)\left(1+\delta^{2}-\beta \bar{\lambda} \rho_{\pi}\right)\left(1-\rho_{\pi}\right)}$, if $\quad \bar{\lambda}<\frac{1+\delta^{2}}{\beta \rho_{\pi}}$;

2) $\alpha-\gamma<\frac{\delta \phi \Theta+\sqrt{\Delta^{\pi}}}{2 \bar{\lambda}^{2}\left(1+\delta^{2}\right)\left(1+\delta^{2}-\beta \bar{\lambda} \rho_{\pi}\right)\left(1-\rho_{\pi}\right)}$, if $\quad \bar{\lambda}>\frac{1+\delta^{2}}{\beta \rho_{\pi}}$.

Taking account of these results, the first group of conditions (i.e. $\Psi>0$ and $\alpha-\gamma>0$ ) which ensure the positive sign for $\frac{\partial^{2} \sigma_{\pi}^{2}}{\partial \sigma_{\varepsilon^{\pi}}^{2} \partial \sigma_{\lambda}^{2}}$ as well as $\frac{\partial^{2} \sigma_{e}^{2}}{\partial \sigma_{\varepsilon^{\pi}}^{2} \partial \sigma_{\lambda}^{2}}$, we obtain the first four conditions in Proposition 3a.

Under the second group of conditions (i.e. $\Psi<0$ and $\alpha-\gamma<0$ ), we can similarly show that to have $\frac{\partial^{2} \sigma_{\pi}^{2}}{\partial \sigma_{\varepsilon^{\pi}}^{2} \partial \sigma_{\lambda}^{2}}$ as well as $\frac{\partial^{2} \sigma_{e}^{2}}{\partial \sigma_{\varepsilon^{\pi}}^{2} \partial \sigma_{\lambda}^{2}}$ is equivalent to find solutions of inequalities in the following two cases.

In the first case, we must have simultaneously the following three inequalities satisfied: $\alpha-\gamma-\frac{\delta \phi \Theta+\sqrt{\Delta^{\pi}}}{2 \bar{\lambda}^{2}\left(1+\delta^{2}\right)\left(1+\delta^{2}-\beta \bar{\lambda}_{\left.\rho_{\pi}\right)\left(1-\rho_{\pi}\right)}\right.}>0, \alpha-\gamma-\frac{\delta \phi \Theta-\sqrt{\Delta^{\pi}}}{2 \bar{\lambda}^{2}\left(1+\delta^{2}\right)\left(1+\delta^{2}-\beta \bar{\lambda} \rho_{\pi}\right)\left(1-\rho_{\pi}\right)}<0$ and $\alpha-\gamma<0$. The first two inequalities can not be true simultaneously if $\bar{\lambda}<\frac{1+\delta^{2}}{\beta \rho_{\pi}}$. If $\bar{\lambda}>\frac{1+\delta^{2}}{\beta \rho_{\pi}}$, we have a solution 
only when we have $\Theta>0$, i.e. $\sigma_{\lambda}^{2}>\max \left\{0, \frac{2 \bar{\lambda}^{2}\left(1-\rho_{\pi}\right)\left(1+\delta^{2}\right)-\beta \bar{\lambda}^{3}\left(1-\rho_{\pi}\right) \rho_{\pi}}{\rho_{\pi}^{2}\left(1+\delta^{2}\right)}\right\}$, then we obtain: $\frac{\delta \phi \Theta+\sqrt{\Delta^{\pi}}}{2 \bar{\lambda}^{2}\left(1+\delta^{2}\right)\left(1+\delta^{2}-\beta \bar{\lambda} \rho_{\pi}\right)\left(1-\rho_{\pi}\right)}<\alpha-\gamma<\frac{\delta \phi \Theta-\sqrt{\Delta^{\pi}}}{2 \bar{\lambda}^{2}\left(1+\delta^{2}\right)\left(1+\delta^{2}-\beta \bar{\lambda} \rho_{\pi}\right)\left(1-\rho_{\pi}\right)}$, which is compatible with the condition $\alpha-\gamma<0$.

In the second case, the following three inequalities must be simultaneously checked: $(\alpha-\gamma)-\frac{\delta \phi \Theta+\sqrt{\Delta^{\pi}}}{2 \bar{\lambda}^{2}\left(1+\delta^{2}\right)\left(1+\delta^{2}-\beta \bar{\lambda} \rho_{\pi}\right)\left(1-\rho_{\pi}\right)}<0, \quad(\alpha-\gamma)-\frac{\delta \phi \Theta-\sqrt{\Delta^{\pi}}}{2 \bar{\lambda}^{2}\left(1+\delta^{2}\right)\left(1+\delta^{2}-\beta \bar{\lambda} \rho_{\pi}\right)\left(1-\rho_{\pi}\right)}>0 \quad$ and $\quad \alpha-\gamma<0 . \quad$ If $\bar{\lambda}>\frac{1+\delta^{2}}{\beta \rho_{\pi}}$, there is not any solution whatever is the sign of $\Theta$. If $\bar{\lambda}<\frac{1+\delta^{2}}{\beta \rho_{\pi}}$ and $\Theta>0$, both of the two first inequalities imply that $\alpha-\gamma>0$ and hence incompatible with the condition $\alpha-\gamma<0$. If $\Theta<0$ (i .e. $0<\sigma_{\lambda}^{2}<\frac{2 \bar{\lambda}^{2}\left(1-\rho_{\pi}\right)\left(1+\delta^{2}\right)-\beta \bar{\lambda}^{3}\left(1-\rho_{\pi}\right) \rho_{\pi}}{\rho_{\pi}^{2}\left(1+\delta^{2}\right)}$ ), we obtain:

$$
\alpha-\gamma>\frac{\delta \phi \Theta-\sqrt{\Delta^{\pi}}}{2 \bar{\lambda}^{2}\left(1+\delta^{2}\right)\left(1+\delta^{2}-\beta \bar{\lambda} \rho_{\pi}\right)\left(1-\rho_{\pi}\right)} \text { and } \alpha-\gamma<\frac{\delta \phi \Theta+\sqrt{\Delta^{\pi}}}{2 \bar{\lambda}^{2}\left(1+\delta^{2}\right)\left(1+\delta^{2}-\beta \bar{\lambda} \rho_{\pi}\right)\left(1-\rho_{\pi}\right)} \text {. } \quad \text { Q.E.D. }
$$

\section{Appendix G: Proof of Proposition 3b}

According to equations (37) and (38) if $\alpha \neq \gamma$, and $\frac{\lambda-\bar{\lambda}}{\lambda} \neq \frac{\left[\left(1+\delta^{2}\right)-\beta \bar{\lambda} \rho_{e}\right]\left[(\alpha-\gamma)\left(1+\delta^{2}\right)+\phi \delta\right]}{\left(1+\delta^{2}\right) \delta \phi \rho_{e}}$, the sign of $\frac{\partial^{2} \sigma_{\pi}^{2}}{\partial \sigma_{\varepsilon}^{2}{ }_{\varepsilon} \partial \sigma_{\lambda}^{2}}$ as well as $\frac{\partial^{2} \sigma_{e}^{2}}{\partial \sigma_{\varepsilon}^{2} e^{\partial \sigma_{\lambda}^{2}}}$ is the same as that of $\Omega(\alpha-\gamma)$. Hence, we study only the conditions under which $\Omega(\alpha-\gamma)$ is positive. We remark that the inequality $\Omega(\alpha-\gamma)>0$ can be checked under two groups of conditions: 1) $\Omega>0$ and $\alpha-\gamma>0$; 2) $\Omega<0$ and $\alpha-\gamma<0$.

Consider now the first group of conditions. To have $\Omega>0$ is equivalent to have:

$$
\left.\left(1+\delta^{2}\right)\left(1-\rho_{e}\right) \bar{\lambda}^{2}\left(1+\delta^{2}-\beta \bar{\lambda} \rho_{e}\right)(\alpha-\gamma)^{2}+\left(1+\delta^{2}\right) \delta \phi 2 \bar{\lambda}^{2}\left(1-\rho_{e}\right)-\rho_{e}^{2} \sigma_{\lambda}^{2}\right](\alpha-\gamma)+\phi^{2} \delta^{2} \bar{\lambda}^{2}\left(1-\rho_{e}\right)>0
$$

Denote: $\Delta^{e}=\left(1+\delta^{2}\right)^{2} \delta^{2} \phi^{2}\left[\rho_{e}^{2} \sigma_{\lambda}^{2}-2 \bar{\lambda}^{2}\left(1-\rho_{e}\right)\right]^{2}-4\left(1+\delta^{2}\right)\left(1-\rho_{e}\right)^{2}\left(1+\delta^{2}-\beta \bar{\lambda} \rho_{e}\right) \phi^{2} \delta^{2} \bar{\lambda}^{4}$.

If $\Delta^{e}>0$, we can solve the above inequality as follows:

$\left\{\alpha-\gamma-\frac{\left(1+\delta^{2}\right) \delta \phi\left[\rho_{e}^{2} \sigma_{\lambda}^{2}-2 \bar{\lambda}^{2}\left(1-\rho_{e}\right)\right]+\sqrt{\Delta^{e}}}{2\left(1+\delta^{2}\right)\left(1-\rho_{e}\right) \bar{\lambda}^{2}\left(1+\delta^{2}-\beta \bar{\lambda} \rho_{e}\right)}\right\}\left\{\alpha-\gamma-\frac{\left(1+\delta^{2}\right) \delta \phi\left[\rho_{e}^{2} \sigma_{\lambda}^{2}-2 \bar{\lambda}^{2}\left(1-\rho_{e}\right)\right]-\sqrt{\Delta^{e}}}{2\left(1+\delta^{2}\right)\left(1-\rho_{e}\right) \bar{\lambda}^{2}\left(1+\delta^{2}-\beta \bar{\lambda} \rho_{e}\right)}\right\}>0$.

We distinguish two cases. In the first case, both expressions in braces must be positive, i.e. $\alpha-\gamma-\frac{\left(1+\delta^{2}\right) \delta \phi\left[\rho_{e}^{2} \sigma_{\lambda}^{2}-2 \bar{\lambda}^{2}\left(1-\rho_{e}\right)\right] \pm \sqrt{\Delta^{e}}}{2\left(1+\delta^{2}\right)\left(1-\rho_{e}\right) \bar{\lambda}^{2}\left(1+\delta^{2}-\beta \bar{\lambda} \rho_{e}\right)}>0$. That yields two conditions: 
1) $\alpha-\gamma>\frac{\left(1+\delta^{2}\right) \delta \phi\left[\rho_{e}^{2} \sigma_{\lambda}^{2}-2 \bar{\lambda}^{2}\left(1-\rho_{e}\right)\right]+\sqrt{\Delta^{e}}}{2\left(1+\delta^{2}\right)\left(1-\rho_{e}\right) \bar{\lambda}^{2}\left(1+\delta^{2}-\beta \bar{\lambda} \rho_{e}\right)}$, if $\bar{\lambda}<\frac{1+\delta^{2}}{\beta \rho_{e}}$;

2) $\alpha-\gamma>\frac{\left(1+\delta^{2}\right) \delta \phi\left[\rho_{e}^{2} \sigma_{\lambda}^{2}-2 \bar{\lambda}^{2}\left(1-\rho_{e}\right)\right]-\sqrt{\Delta^{e}}}{2\left(1+\delta^{2}\right)\left(1-\rho_{e}\right) \bar{\lambda}^{2}\left(1+\delta^{2}-\beta \bar{\lambda} \rho_{e}\right)}$, if $\bar{\lambda}>\frac{1+\delta^{2}}{\beta \rho_{e}}$.

In the second case, both expressions in braces must be negative, i.e. $\alpha-\gamma-\frac{\left(1+\delta^{2}\right) \delta \phi\left[\rho_{e}^{2} \sigma_{\lambda}^{2}-2 \bar{\lambda}^{2}\left(1-\rho_{e}\right)\right] \pm \sqrt{\Delta^{e}}}{2\left(1+\delta^{2}\right)\left(1-\rho_{e}\right) \bar{\lambda}^{2}\left(1+\delta^{2}-\beta \bar{\lambda} \rho_{e}\right)}<0$. That leads to another two conditions:

1) $\alpha-\gamma<\frac{\left(1+\delta^{2}\right) \delta \phi\left[\rho_{e}^{2} \sigma_{\lambda}^{2}-2 \bar{\lambda}^{2}\left(1-\rho_{e}\right)\right]-\sqrt{\Delta^{e}}}{2\left(1+\delta^{2}\right)\left(1-\rho_{e}\right) \bar{\lambda}^{2}\left(1+\delta^{2}-\beta \bar{\lambda} \rho_{e}\right)}$, if $\bar{\lambda}<\frac{1+\delta^{2}}{\beta \rho_{e}}$;

2) $\alpha-\gamma<\frac{\left(1+\delta^{2}\right) \delta \phi\left[\rho_{e}^{2} \sigma_{\lambda}^{2}-2 \bar{\lambda}^{2}\left(1-\rho_{e}\right)\right]+\sqrt{\Delta^{e}}}{2\left(1+\delta^{2}\right)\left(1-\rho_{e}\right) \bar{\lambda}^{2}\left(1+\delta^{2}-\beta \bar{\lambda} \rho_{e}\right)}$, if $\bar{\lambda}>\frac{1+\delta^{2}}{\beta \rho_{e}}$.

Taking account of the condition $\alpha-\gamma>0$, these results lead to the first four conditions of Proposition 3b.

Consider now the second group of conditions with $\Omega<0$ and $\alpha-\gamma<0$. The inequality $\Omega<0$ has two solutions. The first is:

$$
\frac{\left(1+\delta^{2}\right) \delta \phi\left[\rho_{e}^{2} \sigma_{\lambda}^{2}-2 \bar{\lambda}^{2}\left(1-\rho_{e}\right)\right]+\sqrt{\Delta^{e}}}{2\left(1+\delta^{2}\right)\left(1-\rho_{e}\right) \bar{\lambda}^{2}\left(1+\delta^{2}-\beta \bar{\lambda} \rho_{e}\right)}<\alpha-\gamma<\frac{\left(1+\delta^{2}\right) \delta \phi\left[\rho_{e}^{2} \sigma_{\lambda}^{2}-2 \bar{\lambda}^{2}\left(1-\rho_{e}\right)\right]-\sqrt{\Delta^{e}}}{2\left(1+\delta^{2}\right)\left(1-\rho_{e}\right) \bar{\lambda}^{2}\left(1+\delta^{2}-\beta \bar{\lambda} \rho_{e}\right)} .
$$

This is possible when $\bar{\lambda}>\frac{1+\delta^{2}}{\beta \rho_{\pi}}$. Taking account of the condition $\alpha-\gamma<0$, we must also have $\rho_{e}^{2} \sigma_{\lambda}^{2}-2 \bar{\lambda}^{2}\left(1-\rho_{e}\right)>0$, i.e. $\sigma_{\lambda}^{2}>\frac{2 \bar{\lambda}^{2}\left(1-\rho_{e}\right)}{\rho_{e}^{2}}$.

The second solution is:

$$
\frac{\left(1+\delta^{2}\right) \delta \phi\left[\rho_{e}^{2} \sigma_{\lambda}^{2}-2 \bar{\lambda}^{2}\left(1-\rho_{e}\right)\right]-\sqrt{\Delta^{e}}}{2\left(1+\delta^{2}\right)\left(1-\rho_{e}\right) \bar{\lambda}^{2}\left(1+\delta^{2}-\beta \bar{\lambda} \rho_{e}\right)}<\alpha-\gamma<\frac{\left(1+\delta^{2}\right) \delta \phi\left[\rho_{e}^{2} \sigma_{\lambda}^{2}-2 \bar{\lambda}^{2}\left(1-\rho_{e}\right)\right]+\sqrt{\Delta^{e}}}{2\left(1+\delta^{2}\right)\left(1-\rho_{e}\right) \bar{\lambda}^{2}\left(1+\delta^{2}-\beta \bar{\lambda} \rho_{e}\right)}
$$

This is possible when $\bar{\lambda}<\frac{1+\delta^{2}}{\beta \rho_{\pi}}$. Given the condition $\alpha-\gamma<0$, we must also have $\rho_{e}^{2} \sigma_{\lambda}^{2}-2 \bar{\lambda}^{2}\left(1-\rho_{e}\right)<0$, i.e. $\sigma_{\lambda}^{2}<\frac{2 \bar{\lambda}^{2}\left(1-\rho_{e}\right)}{\rho_{e}^{2}}$.

Q.E.D.

\section{References:}

Andolfatto, David and Gomme, Paul (2003), "Monetary Policy Regimes and Beliefs,” International Economic Review, Vol. 44, No. 1, pp. 1-30.

Ball, L. (2000), "Near-Rationality and Inflation in Two Monetary Regimes," NBER Working Paper 7988. Benati, Luca (2008), "Investigating inflation persistence across monetary regimes," Quarterly Journal of Economics, vol. 123(3), pp. 1005-60.

Blanchard, O. J., and J. Gali (2007): "Real Wage Rigidities and the New Keynesian Model," Journal of Money, Credit and Banking, 39(1), pp. 35-65. 
Buiter, W. and I. Jewett (1989), "Staggered Wage Setting and Relative Wage Rigidities: Variations on a Theme of Taylor,” in: Willem Buiter (ed.), Macroeconomic Theory and Stabilization Policy. University of Michigan Press, Ann Arbor, pp. 183-99.

Calvo, G.A., O. Celasun and M. Kumhof (2001), “A Theory of Rational Inflationary Inertia,” in: P. Aghion, R. Frydman, J. Stiglitz and M. Woodford, eds., Knowledge, Information and Expectations in Modern Macroeconomics: In Honor of Edmund S. Phelps. Princeton: Princeton University Press.

Chortareas, G., D. Stasavage and G. Sterne (2002), "Does it pay to be transparent? International evidence from central bank forecasts,” Federal Reserve Bank of St. Louis Review 84, iss. 4, pp. 99-117.

Christiano, L., M. Eichenbaum and C. Evans (2005), "Nominal Rigidities and the Dynamic Effects of a Shock to Monetary Policy,” Journal of Political Economy, vol. 113(1), pp. 1-45.

Clarida, R., J. Gali and M. Gertler (2001), "Optimal monetary policy in open versus closed economies," American Economic Review Papers and Proceedings 91 (2), pp. 248-52.

Clarida, R., J. Gali and M. Gertler (1999), "The Science of Monetary Policy: A New Keynesian Perspective,” Journal of Economic Literature 37, pp. 1661-707.

Corsetti, Giancarlo, Luca Dedola and Sylvain Leduc (2008), "High exchange-rate volatility and low passthrough,” Journal of Monetary Economics 55(6), pp. 1113-28.

Devereux, Michael B. and Charles Engel (2002), "Exchange rate pass-through, exchange rate volatility, and exchange rate disconnect," Journal of Monetary Economics, Vol. 49, Issue 5, pp. 913-40.

Dillén, Hans and Johnny Nilsson (1998), “Transparency, un certainty and monetary policy,” Benjamin Hunt and Adrian Orr (Editors), Monetary policy under uncertainty, Proceedings of a workshop held at the Reserve Bank of New Zealand, June: pp. 204-22.

Dincer, N. Nergiz and Barry Eichengreen (2007), "Central bank transparency: where, why, and with what effects?” NBER Working Paper 13003.

Dittmar, Robert D., William T. Gavin and Finn E. Kydland (2005), "Inflation Persistence and Flexible Prices,” International Economic Review, vol. 46(1), pp. 245-61.

Dornbusch, R. (1987), “Exchange rates and prices,” American Economic Review 77, pp. 93-106.

Krugman, P. (1987), "Pricing to market when the exchange rate changes,” In: Arndt, S., Richardson, J. (Eds.), Real-Financial Linkages among Open Economies. MIT Press, Cambridge.

Eijffinger, S.C.W. and P.M. Geraats (2006), “How transparent are central banks?” European Journal of Political Economy 22, pp. 1-21.

Eijffinger, Sylvester C.W., Lex Hoogduin and Carin A.B. van der Cruijsen (2008), "Optimal Central Bank Transparency,” CEPR DP6889.

Erceg, C. and A. Levin (2003), "Imperfect Credibility and Inflation Persistence," Journal of Monetary Economics, Vol. 50, Issue 4, pp. 915-44.

Fuhrer, J. (2000), "Habit Formation in Consumption and its Implications for Monetary Policy Models," American Economic Review 90, pp. 367-90.

Fuhrer, J. and G. Moore (1995), “Inflation Persistence,” Quarterly Journal of Economics 110, pp. 127-59.

Galí, J. and T. Monacelli (2005), "Monetary policy and exchange rate volatility in a small open economy,” Review of Economic Studies, 2005, vol. 72, issue 3, pp. 707-34.

Gaspar, Vítor, Frank Smets and David Vestin (2006), “Adaptive learning, persistence, and optimal monetary policy,” ECB Working Paper Series No. 644.

Geraats, Petra (2002), “Central Bank Transparency,” The Economic Journal 112, pp. 532-65.

Goldberg, P. and M. Knetter (1997), “Goods prices and exchange rates: what have we learned?” Journal of Economic Literature 35, pp. 1243-72.

Goodfriend, M. and R.G. King (1997), “The new neoclassical synthesis and the role of monetary policy,” NBER Macroeconomics Annual, pp. 231-83.

Goodfriend, M. and R. King (2001), “The Case for Price Stability,” NBER Working Paper 8423.

Huh, C., Lansing, K. (2000), "Expectations, credibility and disinflation in a small macroeconomic model," Journal of Economics and Business 51, pp. 51-86.

Ireland, Peter N. (2000), "Expectations, Credibility, and Time-Consistent Monetary Policy," Macroeconomic Dynamics 4, pp. 448-66. 
Ireland, Peter N. (2004), “A Method for Taking Models to the Data,” Journal of Economic Dynamics \& Control 28, pp. 1205-26.

Kenneth N. Kuttner and Adam S. Posen (2001), "Inflation, Monetary Transparency, and G3 Exchange Rate Volatility,” in Balling, Hennessy and Hochreiter (eds.), Adapting to Financial Globalisation, London: Routledge.

Krugman, Paul R. (1987), "Pricing-to-MarketWhen the Exchange Rate Changes,” in S.W. Arndt and J.D. Richardson, eds., Real Financial Linkages Among Open Economies. Cambridge Mass: MIT Press.

Leitemo, Kai and Ulf Söderström (2008), "Robust Monetary Policy in a Small Open Economy,” Journal of Economic Dynamics and Control, vol. 32(10), pp. 3218-52.

Mankiw, N.G. and R. Reis (2001), "Sticky Information Versus Sticky Prices: A Proposal to Replace the New Keynesian Phillips Curve,” NBER Working Paper 8290.

McCallum, Bennet T. (1983), "On non-uniqueness in rational expectations models: An attempt at perspective,” Journal of Monetary Economics, 11(2), pp. 139-68.

Mennon, J. (1995), “Exchange rate pass-through,” Journal of Economic Surveys 9 (2), pp. 197-231.

Nelson, E. (1998), "Sluggish Inflation and Optimising Models of the Business Cycle," Journal of Monetary Economics 42, pp. 303-22.

Pivetta, F. and R. Reis (2007), “The Persistence of Inflation in the United States,” Journal of Economic Dynamics \& Control 31, pp. 1326-58.

Roberts, J. (1998), "Inflation Expectations and the Transmission of Monetary Policy," Finance and Economics Discussion Paper no. 98-43. Washington, D.C.: Board of Governors of the Federal Reserve System.

Rotemberg, J.J. and M. Woodford (1997), "An optimization-based econometric framework for the evaluation of monetary policy,” in B.S. Bernanke and J.J. Rotemberg (eds.), NBER Macroeconomic Annual, The MIT Press.

Sargent, T. (1999), The Conquest of American Inflation. Princeton University Press.

Sims, C. (2001), “Implications of Rational Inattention,” Manuscript, Princeton University.

Smets, Frank and Raf Wouters (2002), "Openness, imperfect exchange rate pass-through and monetary policy,” Journal of Monetary Economics, Vol. 49, Issue 5, pp. 947-81.

Steinsson, J. (2003), “Optimal Monetary Policy in an Economy with Inflation Persistence,” Journal of Monetary Economics 50, pp. 1425-56.

Stock, J. (2001), "Comment on Evolving Post-World War II U.S. Inflation Dynamics," NBER Macroeconomics Annual 2001, pp. 379-87.

Svensson, Lars E.O. (2002), "Inflation Targeting: Should It Be Modeled as an Instrument Rule or a Targeting Rule?” European Economic Review 46, pp. 771-80.

Taylor, J.B. (1983), “Union wage settlements during a disinflation,” American Economic Review 73, pp. 981-83.

Van der Cruijsen, Carin and Maria Demertzis (2007), “The impact of central bank transparency on inflation expectations,” European Journal of Political Economy, vol. 23, issue 1, pp. 51-66.

Westelius, N.J. (2005), “Discretionary monetary policy and inflation persistence,” Journal of Monetary Economics 52, pp. 477-96.

Woodford, M. (2001), "Imperfect Common Knowledge and the Effects of Monetary Policy,” NBER Working Paper 8673. 